\title{
Die syro-armenischen und die syro-koptischen Bibelcitate aus den Scholien des Barhebräus.
}

\author{
Von Dr. J. Goettsberger.
}

Die folgenden Seiten wollen der Ausbeute des Scholienwerkes von Barhebräus dienen, das auch in dieser Zeitschrift ${ }^{x}$ nicht unbekannt ist. Die textkritische Veranlagung dieses berühmten syrischen Schriftstellers hat seinen Scholien zur hl. Schrift eine grosse Bedeutung für verschiedene Bibelübersetzungen gegeben. Für die Peschittho liefert Barhebräus manche Varianten; für die syrohexaplarische Version mit ihren Randlesungen (Aquila, Symmachus, Theodotion, Quinta, Sexta, Hebräer, Samaritaner) bietet er teilweise Fragmente zu verlorenen Büchern, teilweise auch Ergänzungen und Correcturen zum jetzigen Textbestand. Nicht so bedeutsam, aber wohl einer Besprechung wert sind seine Citate aus der armenischen und koptischen Bibelübersetzung.

\section{Die syro-armenischen Bibelcitate.}

Die Syrer standen mit den Armeniern in naher geographischer Verbindung, so dass sich ein gegenseitiger literarischer Verkehr leicht entwickeln konnte. Bei unserem Schriftsteller kamen dazu noch andere Gründe. Ihn brachte Heimat (Melitene in Kleinarmenien) und Wirkungskreis (als "Maphrian des Ostens") in enge Berührung mit diesem Volke. So kann es nicht auffallen, dass Barhebräus neben anderen Schriftexten auch zur armenischen Bibelübersetzung

I Vgl. 1896 p. $249-64 ; 1898$, p. 177-196. 
griff, um ein möglichst sicheres Fundament für seine Schrifterklärung ${ }^{2} \mathrm{zu}$ finden.

$\mathrm{Da}$ bisher nur einige wenige Stellen von den syroarmenischen Citaten des Barhebräus verwertet worden sind, ${ }^{2}$ so sollen nunmehr alle diese Anführungen des Barhebräus gesondert zusammengestellt, mit dem jetzt vorliegenden armenischen Bibeltexte verglichen und näher erörtert werden. Als Grundlage für den Scholientext des Barhebräus dienen die Ausgaben der einzelnen Scholienteile, besonders die Scholien zu den Psalmen, herausgegeben von Lagarde, 3 verglichen und ergänzt nach der zuletzt erworbenen wichtigsten Handschrift der Scholien. 4 Für die armenische Bibelübersetzung sind die 2 hauptsächlichsten Venediger Ausgaben von 1805 (mit Varianten) und 1860 beigezogen worden. 5 Die griechische Vorlage ist ab und $z u$ nach dem Handschriften-Material von Swete ${ }^{6}$ verwendet worden. Zur Anlage des folgenden Verzeichnisses ist zu bemerken: zuerst steht das syro-armenische Citat des Barhebräus, in Klammern allenfalls die Lesung aus Peschittho $(=\mathrm{P})$ oder Syrohexapla $(=\mathrm{Sh})$, die bestätigt oder corrigiert werden soll; ein folgendes $=$ kennzeichnet Übereinstimmung, ein | Differenz mit der armenischen Übersetzung, die in armenischer Schrift

× Das erklärt er selbst in der Einleitung zu den Scholien als Ziel seiner textkritischen Bemerkungen.

2 Vgl. Walton, Polyglotta, tom. VI: Excerpta paucula ex Scholiis Gregorii Syri; Wiseman, Horae syriacae p. 142 ss.; Rhode, Gregorii Barhebraei scholia in Ps. V. et XVIII. p. 74 ss.; Eichhorn, Repertorium für morgenlaendische Litteratur, XIII. p. I84 ff.

3 Lagarde P., Praetermissorum libri duo, Gottingae 1879 pp. 97244. - Auch O. F. Tullberg hat die Scholien zu Ps. I. 2. 22 herausgegeben (Upsalae I842).

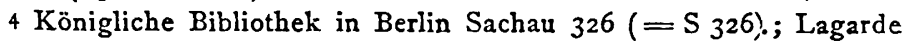
konnte nur 2 Hsn vergleichen.

5 Abkürzungen: 1805, 1805 Var. und 1860.

6 The Old Testament in Greek, II (sec. ed.); die dort angewendeten Abkürzungen sind beibehalten. 
mit deutscher Übertragung folgt; Fussnoten sollen das gegenseitige Verhältnis näher angeben.

Wiseman hat (Horae syr. p. 145) bemerkt ${ }^{x}$, Barhebräus gebe armenische Lesungen nur zu den Psalmen. Jedoch citiert Barhebräus eine vereinzelte Lesung auch zu

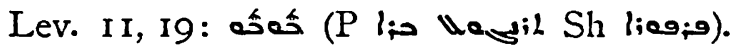

Es frägt sich, ob es sich hierbei um Citate aus den betreffenden Übersetzungen (auch arabisch wird das Wort wiedergegeben) oder um Erklärungen aus den angegebenen Sprachen handelt. In der Veröffentlichung der syrohexaplarischen Fragmente zu Leviticus hat G. Kerber ${ }^{2}$ das Citat Sh lo: ausgelassen, weil er es offenbar nicht als dem syrohexaplarischen Bibeltexte entnommen betrachtet. Dem entsprechend könnte auch das armenische und das arabische Citat (auch diese Bibelübersetzung wird an vereinzelten Stellen beigezogen) als blos sprachlich-lexikalische Parallele gelten. Aber zweifellos haben wir in diesen Anführungen Citate aus der syrohexaplarischen, arabischen und armenischen Bibelübersetzung zu suchen und zwar aus folgenden Gründen: I) unter a oder Le (hier حس) ist in der Regel 3 bei Barhebräus die Sh zu verstehen; 2) in der griechischen Vorlage steht zwar das dem syrischen

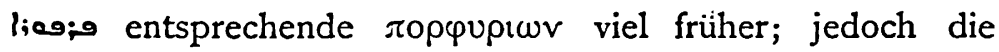
Stellung der einzelnen Worte im Griechischen ist durchaus nicht feststehend und massgebend; in der Parallelstelle 4

I Ihm folgte Rhode, 1; c., p. 74.

2 Vgl. diese Zeitschrift 1896 p. 253.

3 a des Barhebrāus zu 2 Sam. 3, 29 ergānzt das Lexikon des Bar Bahlul (herausgegeben von R. Duval) p. $103^{8} \mathrm{zu}$ we $=$ Johannes, jedoch kaum mit Recht, was anderwärts zu untersuchen wäre. Zu Js. 48, I versteht Barnebräus darunter die griechische Sprache, nicht den syrohexaplarischen Bibeltext.

4 Der hebräische Text und die Peschittho sprechen dafür, dass beide Stellen in $\mathrm{Zahl}$ und Ordnung der Worte sich ursprünglich deckten. 
Deut. 14, 17 bietet die Hs. B bei Swete gerade hier das rop'puptwr; 3) die arabische Bibelubersetzung hat wirklich das Wort هُ20 das Barhebräus aus dem Arabischen beizieht, an der gleichen Stelle; also ist dieses Citat des Barhebräus als Bibelcitat aufzufassen. Diese Gründe sprechen auch für den biblischen Ursprung des armenischen Citates zu Lev. I I, 19. Ausser Zweifel gestellt wird diese Thatsache, wenn wir beachten, dass das rätselhafte ${ }^{x}$ sich als ein Missverständnis ${ }^{2}$ einer armenischen Vorlage begreift. In der armenischen Bibel steht an der Stelle $\boldsymbol{J}^{n u} \boldsymbol{l}^{n \boldsymbol{u} l}$, von einem Syrer von rechts nach links gelesen $=$ bobo. 3 Hiedurch ist ein armenisches Citat auch ausserhalb der Psalmen festgestellt.

Nur ein Citat findet sich, um dies vorauszunehmen, im

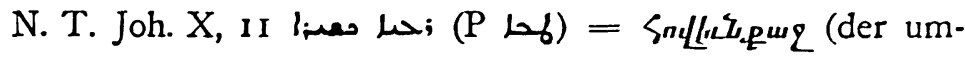
sichtige Hirte). Die übrigen Citate finden sich im Commentar zu den Psalmen.

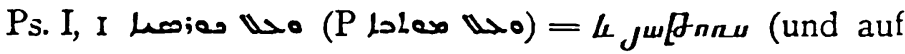
die Sitze oder Throne ${ }^{4}$ ).

I Das Wort Lagarde, Praetermissorum libri duo p. 731.47 wohl, aber in ganz anderer Bedeutung (= tumor bubonum, morbi genus).

2 Wie sehr Missverständnisse dem Barhebräus oder seinem Gewährsmanne zuzutrauen sind, wird das folgende Verzeichnis lehren. Vergl. 2u S. 123. Anm. 3.

3 Auch $\mathrm{BB}(=\mathrm{Bar}$ Bahlul) p. 213 s. v. Lesl und P. S. (Payne Smith, Thes. syr.) p. 2388 s. v. liog;9 citieren diese Stelle, offenbar aus Barhebräus zu Lev. II, 19, aber mechanisch und fehlerhaft, wie eine einfache Vergleichung ergiebt.

4 Nach Novum Lexicon linguae Armeniacae, Venetiis 1836 (= Lex.)

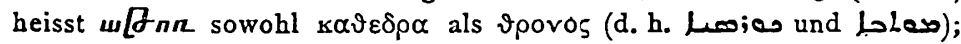
hätte also Barhebräus selbst unmittelbar den armenischen Text ein. gesehen, so hätte er keinen Grund gehabt, hier für das armenische sinngleiche Wort eine von $P$ verschiedene syrische Übersetzung zu wählen. Jedoch soll nach einer gütigen Mitteilung des P. Wartan Melchizedek, Mechitaristen in Wien, der Gebrauch für "Thron“ überwiegend sein. 


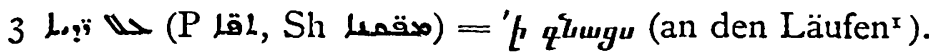

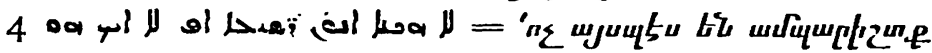

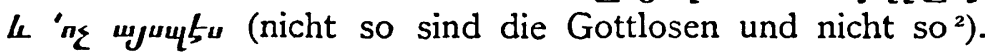

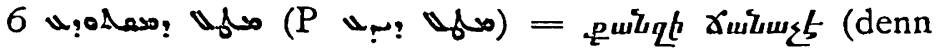
er weiss 3 ).

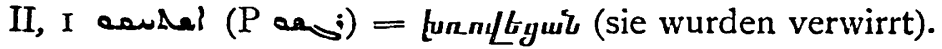

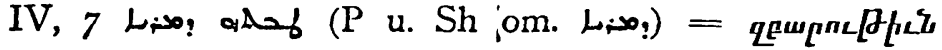
untum ל (1860, I 805 Var. die Güte des Herrn4).

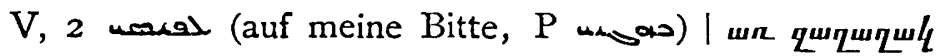
15 (auf mein Geschrei5).

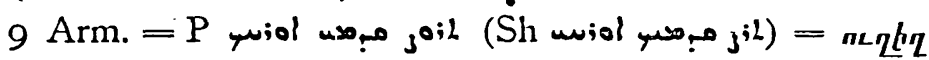

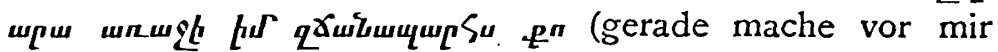
deine Wege).

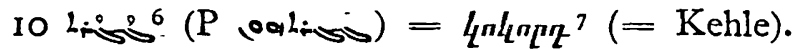

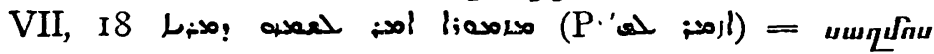
wumglg whnцul untwm ל (einen Psalm werde ich sagen dem Namen des Herrn).

I Lex. q quug steht auch an andern Stellen für $\delta \imath \varepsilon \zeta ั 0 \delta \circ 5 ;$ also gilt die gleiche Bemerkung wie S. 104, Anm. 4.

- 2 oa إم die wörtliche Übersetzung von $u J^{u}=0$ und $45 u$

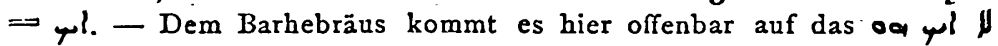
an; hätte er unmittelbar aus dem Armenischen geschöpft, so wäre kein

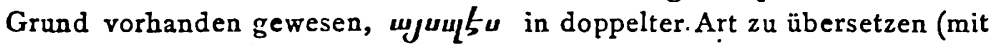
Lea und oa (l.) - Eichhọrn, Repertorium für morgenländische Literatur XIII, 184 bemerkt: oủx oútws fehle zum zweitenmale in der armenischen Übersetzung, was nach Obigem nicht richtig ist.

3 Die Differenz der beiden Formen scheint eine innersyrische zu sein, beide können das armenische Wort wiedergeben.

4 Der Text der Ausgaje von 1805 lässt, wie die griechischen Hsn bei Swete, den Genetiv aus.

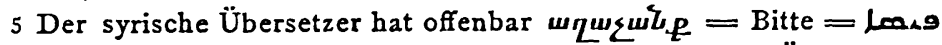
mit dem im Texte stehenden richtigen Worte wegen der Ähnlichkeit verwechselt.

6 Rhode und Lagarde bieten _Lr

7 Aus der Form (ohne Suffix) und der Punktation (syr. IL ist ersichtlich, dass wir es hier mit einer Transscription des armenischen Wortes ins Syrische zu thun haben. 
VIII, 5 صئه $=$ P (nestor.

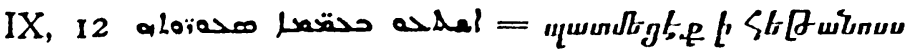

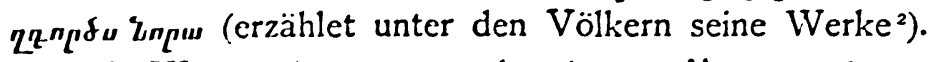

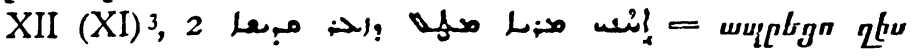

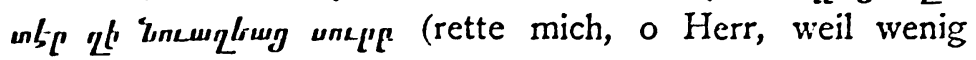
geworden ist der Heilige).

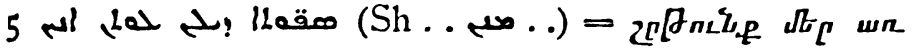

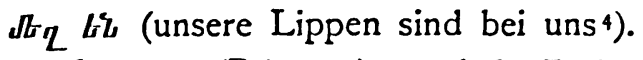

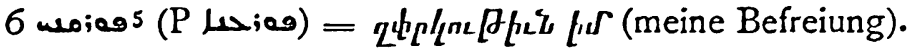

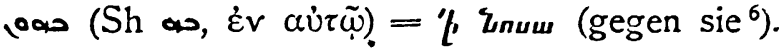

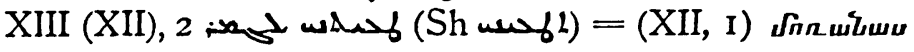
qtiu ' $t$ шuшn (vergissest du mich bis zum Ende).

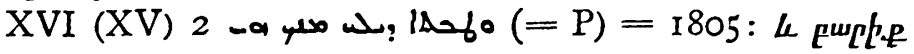

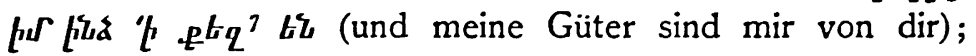
1805 Var. und $1860=$ LXX (und meine Güter sind dir nicht in irgend etwas nötig $\left.{ }^{8}\right)$.

I Barhebrāus hält das Neutr. $\tau$ i für richtig mit Berufung auf Sh; unter den griechischen Hsn bei Swete hat A ri5.

2 In odal und ILo;cseichen P und Sh von Arm. ab, ohne dass jedoch diese beiden Worte die einzige Übersetzung des armenischen Textes bildeten; er könnte gerade so gut mit den Worten von $P$ und $S h$ wiedergegeben sein.

3 Die Psalmennummerierung in Klammern entspricht der griechischen Zählung, die armenische Bibel stimmt, wo es nicht anders bemerkt ist, damit überein.

4 Arm und Sh haben die Präposition $\pi \alpha \rho \alpha$ verschieden aufgefasst.

5 Lagarde zieht noch AL zum armenischen Citat (ebenso Schröter, ZDMG XXIX, 282); aber Barhebräus wiederholt dieses Wort aus der $P$, weil er eine Variante aus $S h$ dazu anführt.

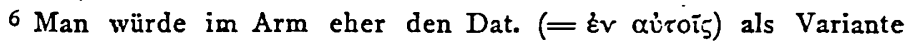
erwarten.

$7 p^{t}\{$ ist Druckfehler.

8 Hier fügt Barhebräus die bekannte (vergl. Eb. Nestle in „Urtext und Übersetzungen der Bibel"s Sep. Abdr. aus PREncycl. p. 157) Bemerkung an: „Und hieraus ist ersichtlich (م, (محا), dass, wenn auch die Armenier aus dem Griechischen übersetzten, sie doch hinwiederum ihre Handschriften mit dem Syrischen verglichen und hie und da mit ihm 


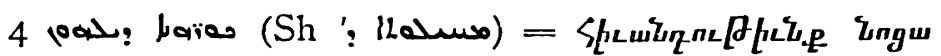
(ihre Krankheiten ${ }^{x}$ ).

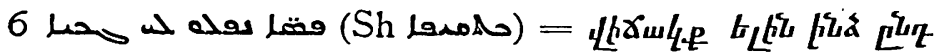

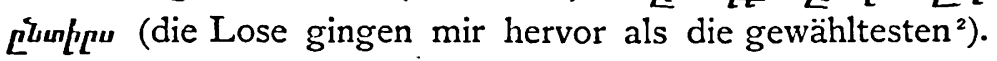

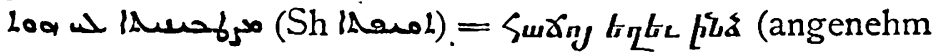
ist sie $\mathrm{mir}^{3}$ ).

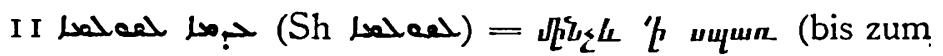
Ende 4).

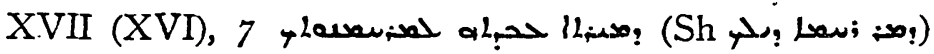

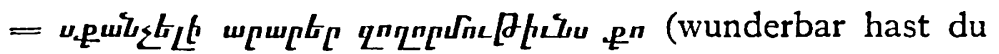
gemacht deine Erbarmungen).

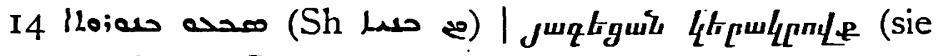
sättigen sich mit Speisen 5).

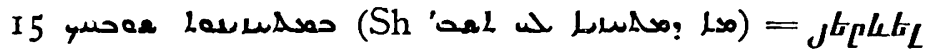
twunum png (beim Erscheinen deines Ruhmes).

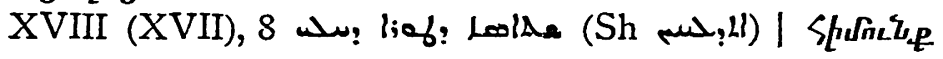

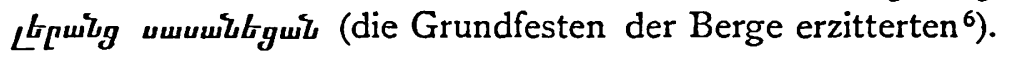

in Übereinstimmung brachten.“ Manche erklären diese Stelle dahin, dass Barhebräus nur eine Mutmassung ausspreche auf Grund dieser zufälirgen Übereinstimmung (Wiseman, 1. c. p. 142; Cornely, Introductio I, 3874 ; Vigouroux, Dictionnaire de la Bible I, IOII). Wenn auch nicht, wie Valton, Polygl. tom. I. p. 91 glaubt, deuten will, so scheint doch Barhebräus hier nur etwas bestätigt zu finden, was sonst allgemeine Anschauung war, also nicht eine rein persōnliche Vermutung auszusprechën.

I Sh und Arm variieren nur mit synonymen Worten.

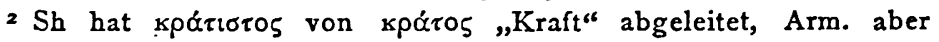
richtig als Superlativ von $\dot{\alpha} \alpha \vartheta \circ 5$ gefasst, ebenso in I Reg. 15, 15; W ist wörtliche Wiedergabe des Armenischen. - Die Differenz von

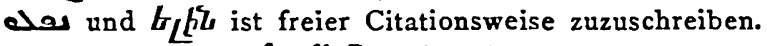

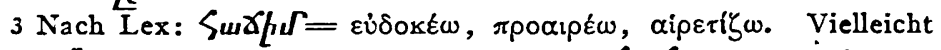

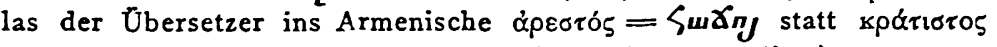
der LXX.

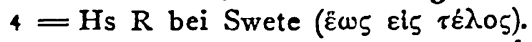

5 Die sonderbare syrische Wiedergabe des armenischen $l^{t}\left[m L_{L} / L\right.$

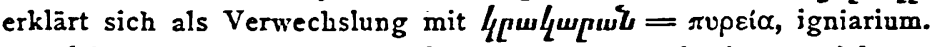

6 Barhebräus ,die Grundfesten der Berge fürchteten sich." - Es ist irgend ein Missverständnis nicht ausgeschlossen. 


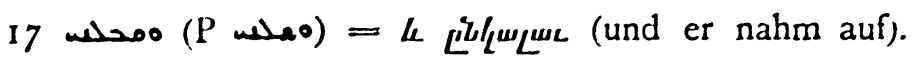

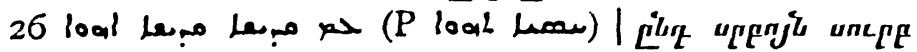
Ir higlsu (mit dern Heiligen wirst du heilig sein ${ }^{3}$ ).

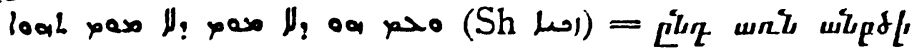
whplif liqliglru (gegen den Tadellosen wirst du tadellos sein).

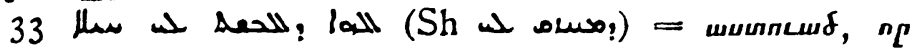

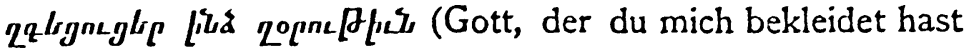
mit Stärke ${ }^{2}$ ).

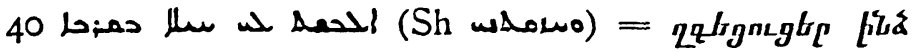

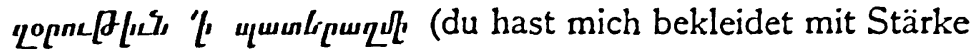
im Kriege).

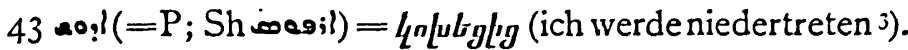

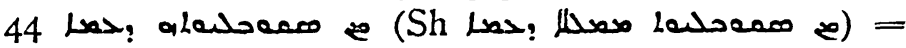

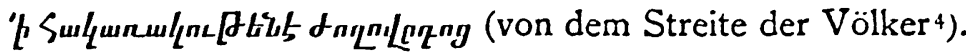

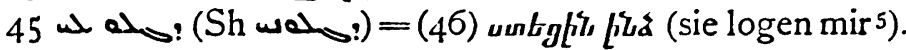

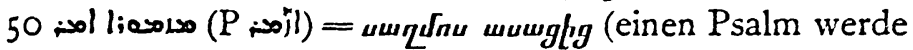
ich sagen $\left.{ }^{6}\right)$.

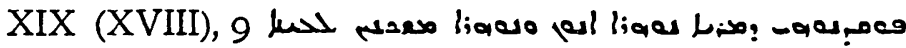

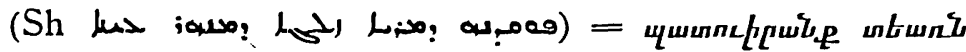
Ly und Licht gewähren sie den Augen).

I Schon Rhode, 1. c. p. 83 not. 54 verzeichnet diesen Unterschied.

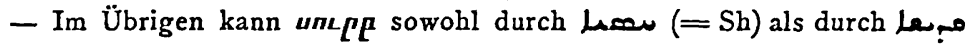

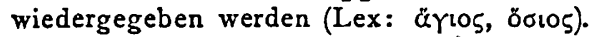

2 Rhode, 1. c. p. $54^{*}$ bemerkt, die Amsterdamer Ausgabe der armenischen Bibel habe: induit.

3 Die verschiedenen syrischen Worte sind synonym.

4 Auch im armenischen Worte sind die beiden Bestandteile dंrq-

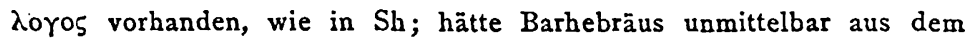
Armenischen geschöpft, so hätte er die armenische Leseart nicht von Sh verschieden wiederzugeben gebraucht. - حما Accommodierung an $\mathrm{Sh}$ u. $\mathrm{P}$ sein.

5 Der Unterschied zwischen Sh und dem Syrisch-Armenischen ist blos formell; vergl. Noeldeke, Syr. Gr. 2 \& 292.

6 Vergl. VII, 18. 


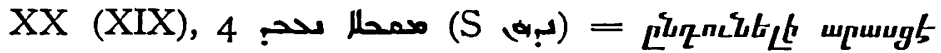
(angenehm wird er machen).

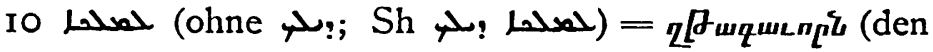
König ${ }^{\mathrm{I}}$ ).

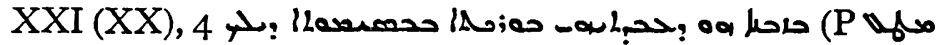

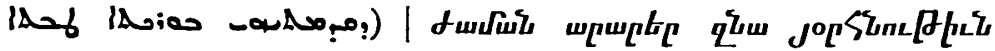

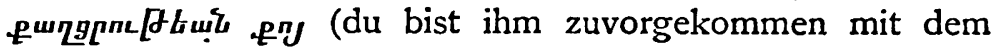
Segen deiner Süssigkeit ${ }^{2}$ ).

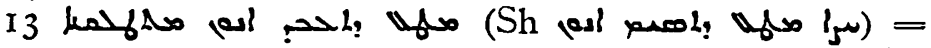

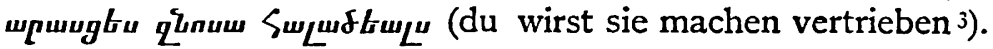

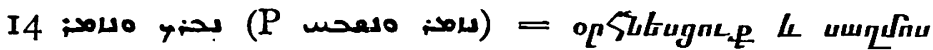
шuшugnце (wir werden segnen und Psalmen sagen).

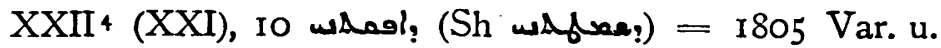
I860: $\pi$ S Sultr q qu (der du mich herausgebracht hast; I805 Switu $=\operatorname{der}$ du mich zusammengewoben hast5).

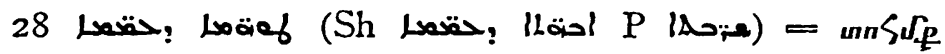
wquilg (Stämme der Völker ${ }^{6}$ ).

- I So auch einige Hsn bei Swete.

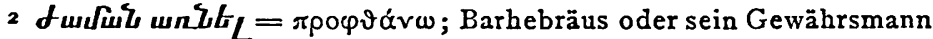

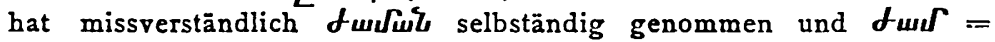
xpóvo Zeit übersetzt; infolge dessen musste auch das Übrige etwas modifiziert werden, um einen leidlichen Sinn zu gewinnen („zur Zeit, da du ihm bereitet hast Segen in deiner Süssigkeit").

3 : Uso, das der armenische Text nicht bietet, lässt sich als freie Accommodation des Citates an Sh erklären, wie es bei Barhebräus öfter vorkommt. Der armenische Übersetzer scheint die griechische Vorlage

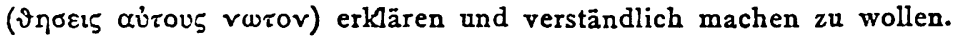

4 Die Excerpta paucula von Loftusius in Walton, tom. VI bieten zu XXII, I eine Lesung in armenischer Schrift und Sprache; .die Ausgaben von Lagarde, Tullberg und die Hs. S 326 enthalten nichts dergleichen.

5 Die Leseart der Bibelausgabe von 1805 ist ein Versehen, aus dem Armenischen leicht erklärbar.

6 Barhebrāus fügt hinzu: ba o lo ,und wie das syrische Wort spricht er dieses Substantiv aus"d. h. die 


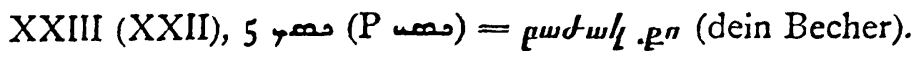

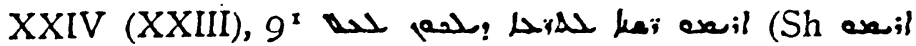

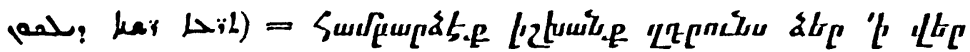
(hebet, Fürsten, euere Thore auf).

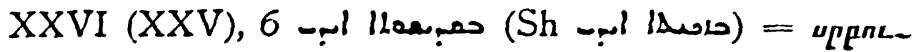

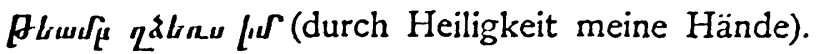

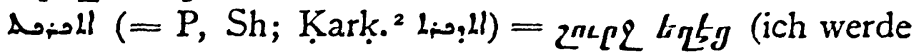
herum sein).

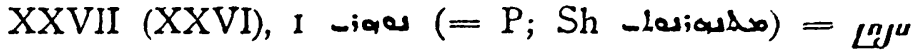
bis (mein Licht 3 ).

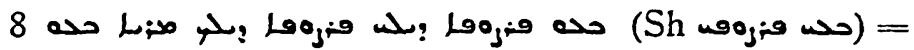

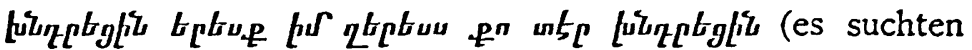
meine Gesichte, deine Gesichte, Herr, suchten sie ${ }^{4}$ ).

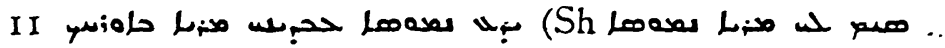

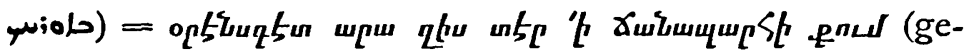
setzekundig mache mich, Herr, auf deinem Wege).

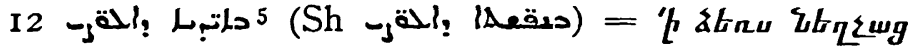
lıfng (in die Hände meiner Unterdrücker).

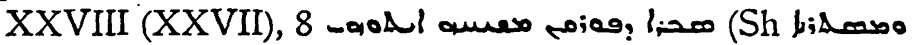

armenische Übersetzung hat nicht bloss ein Wort, das Loo of bedeutet, sondern ein Wort, das auch lautlich (ح) ihm gleicht. Tullberg ibbersetzt: „sed secundum consuetudinem sermonis syriaci corrupto utitur hoc vocabulo," als ob hier gemeint sei, dass ein armenisches Wort nach der syrischen Sprechweise verderbt sei. Diese Auffassung scheint mir unrichtig zu sein.

I Lagarde unrichtig v. Io.

$2=$ Traditio Karkaphensis, d. h. Hsn einer Schule syrischer Punktatoren.

3 Nach Lex. bedeutet $/ n J^{\boldsymbol{\nu}} \varphi \bar{\omega} \zeta$ und $\varphi \omega \tau \sigma \mu$ ó ; also scheint auch diese Variante anzudeuten, dass der Armenier dem Barhebräus schon syrisch vorlag.

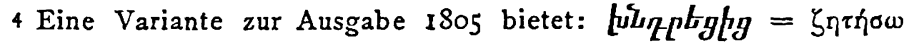
(ich werde suchen).

5 So in Hs. S 326 ; Lagarde hat dieses Citat nicht. 


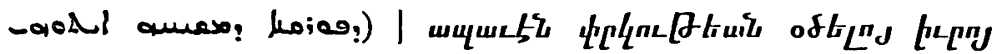
(der Schützer der Rettung seines Gesalbten $=\mathrm{Sh}^{\mathrm{x}}$ ).

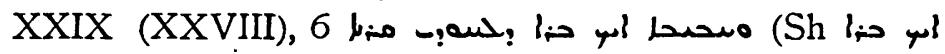

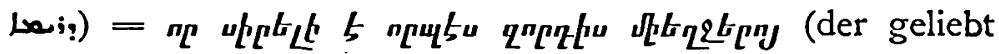
ist wie das Junge des Einhorns ${ }^{2}$ ).

8 lig ("statt $\infty$ des Griechen setzt er " ") (Sh $\infty$,h)

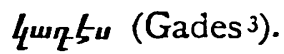

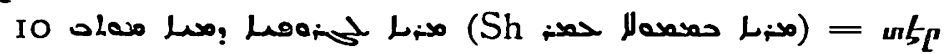

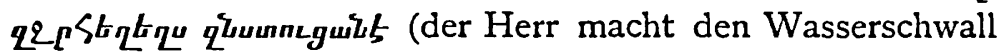
sich setzen 4$)$.

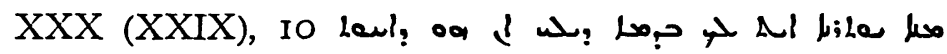

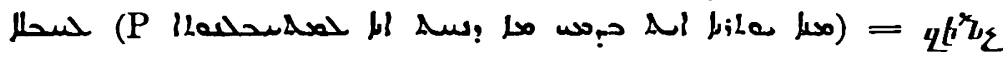

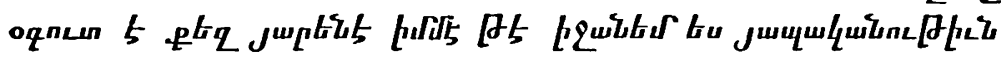
(welcher Vorteil ist dir in meinem Blute, wenn ich hinabsteige in das Verderben).

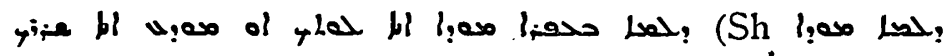

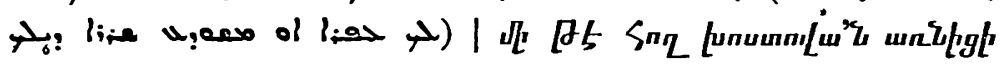

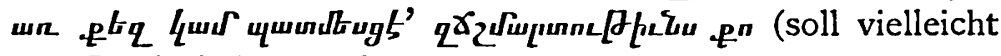
der Staub bekennend gemacht werden zu dir oder soll er erzählen deine Wahrheiten $=\mathrm{Sh}^{5}$ ).

I محتץ setzt ein $\varepsilon \lambda \pi \zeta \zeta \omega$ voraus; vielleicht beruht die syrische

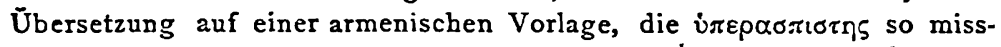
verstanden hat. Mōglicherweise ist ursprünglich im Syrischen

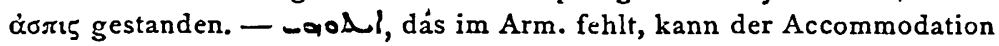
an $\mathrm{Sh}$ entstammt sein.

2 Varianten in der Ausgabe von 1805 betreffen nur andere Genetivbildung. - Die Differenz محسم o kann der Accommodation an Sh zugeschrieben werden.

3 Diese Leseart findet sich nirgends vertreten, bemerkt P. Wartan. Nicht unwahrscheinlich haben wir es hier mit einem Missverständnis zu thun; in der Kurrentschrift sehen sich $Z$ (oft $=1)$ u. $q(=2)$ ganz gleich.

4 مس 4 مس ( $S t_{q} t_{Z}=$ karakivenós = L90; $)$.

5 Es wāre nicht unmöglich, dass 3. sg. fut pass. wn 2 /.gh verwechselt wurde mit 1. sg. aor. I., eine Form. die ebenfalls auf $-g h$ endigen würde. 


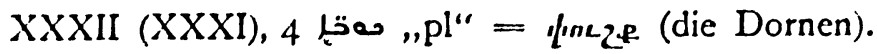

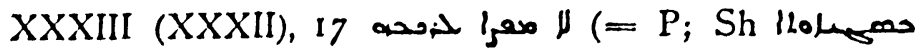

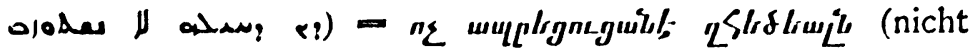
rettet es scinen Reiter).

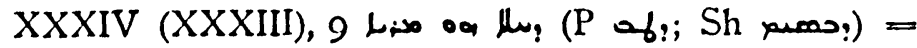

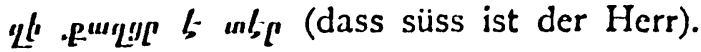

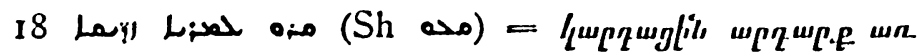
anfsp (es riefen die Gerechten zum Herrn ${ }^{2}$ ).

XXXV (XXXIV), أهم (Sh Sall (ziche sc. dein Schwert).

I7 aol (P yoal) = "l lumg wlu (bringe heraus).

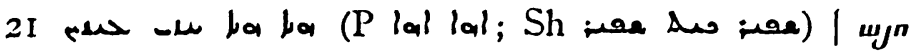

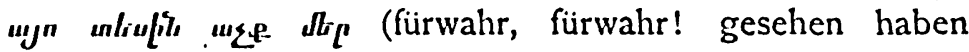
unsere Augen ${ }^{2}$ ).

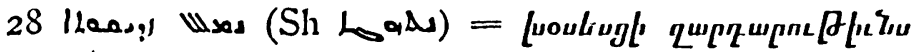
en (wird reden deine Gerechtigkeit ${ }^{3}$ ).

XXXVI (XXXV), 7 lall liaf or "ohne " (Sh ladl:) = Llichit.p munnцud (Berge, Gott).

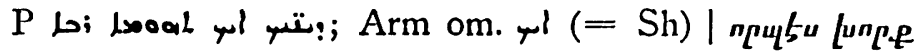

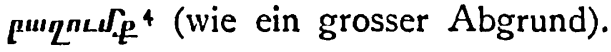

II 1 i!l (Sh wods) = funqliw (lass aufgehen).

Sonst müsste bei Barhebräus das Zeugnis für eine neue Lesart gefunden werden: "soll ich etwa im Staube zu dir bekennen oder verkündigen deine Wahrheit?"

I Lagarde trennt übrigens Lمـ! durch einen Punkt; darnach könnte die Leseart imperativisch gemeint sein, also eine neue Variante des armenischen Textes bieten.

2 Barhebräus oder sein Gewährsmann haben statt ujn (= = $u ̛ \gamma \varepsilon$ ) verlesen $\boldsymbol{u} \boldsymbol{u}$ (= ba dieses).

$3 \mathrm{Zu} \gamma \lambda \omega \sigma \sigma \alpha$ konnte der armenische Übersetzer $\mu \varepsilon \lambda \varepsilon \tau i j \sigma \varepsilon l$ nicht reimen.

4 So ISo5 Var.; im Text hat die Ausgabe von 1 So5 den acc. ( $\rightarrow$ 1860). - Auch einzelne Hsn bei Swete lassen wort aus. 


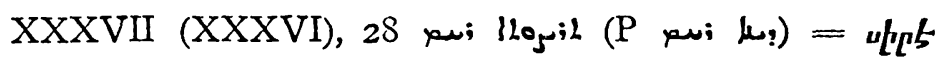

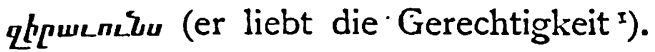

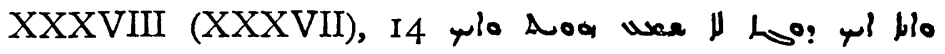

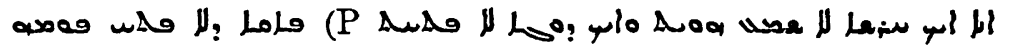

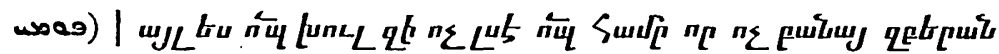
hLp (aber ich bin wie ein Tauber, denn nicht hört er, wie ein Stummer, der nicht öffnet seinen Mund ${ }^{2}$ ).

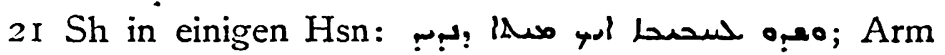
om. $(=\mathrm{P})$; wird bestätigt durch die Ausgaben.

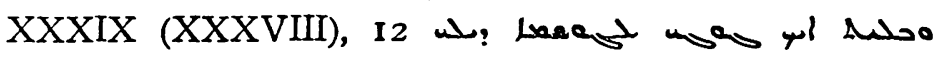

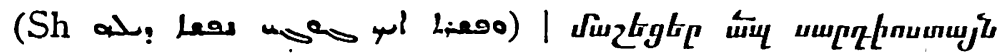

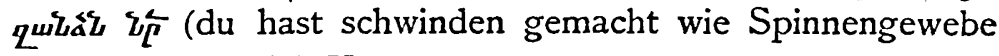
seine Seele $\left.=\mathrm{LXX}^{3}\right)$.

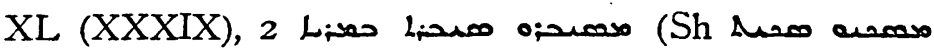

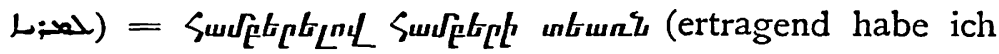
ertragen den Herrn 4 ).

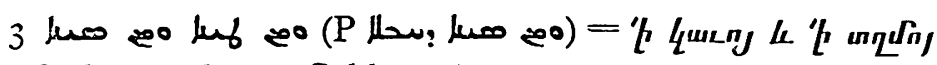
(von Lehm und von Schlamm).

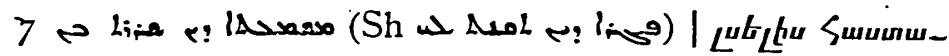
- untghtr lís (das Gehör befestigtest du mirs).

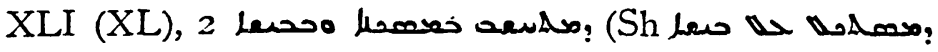

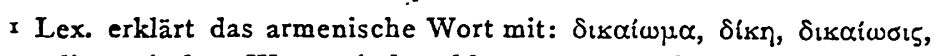
кpip $\alpha$; die syrischen Worte sind wohl synonym $z$ u fassen.

2 Barhebräus kommt es auf "taub" und "stumm" an, die in $P$ an falscher Stelle stehen; darum können die Differenzen mit dem armenischen Texte auf Accommodation an Sh zurückgeführt werden.

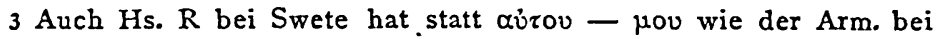
Barhebrāus. Laey würde dem Bestreben entsprechen, unpassende Vorstellungen (wie hier: Hinschwinden der Seele) fern zu halten, wie es sich offer im Armenischen zeigt; vglo $z$ XXXV, 28.

4 der Sh und هصح 4 des Arm. dürfen hier wohl als synonym betrachtet werden.

5 Hier liegt eine neue Leseart bei Barhebrāus vor: „das Gehör nun befestigtest du in uns."

Zeitschrift E. d. alttest. Wiss. Jahrg. 2x. I. 190x. 


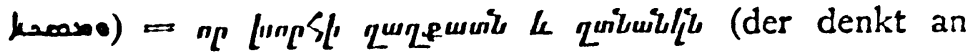
den Armen und Elenden).

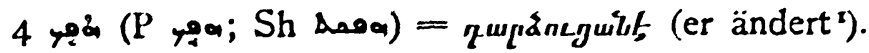

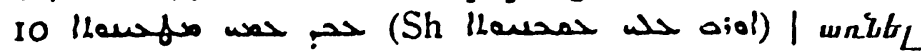
filus luwahaclflı\& (zu bereiten mir Täuschung; gegen Barh: an $\mathrm{mir}$ ).

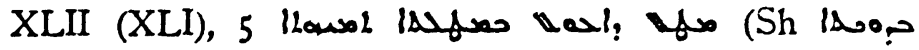

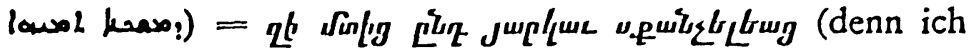
werde eintreten unter das wunderbare Dach).

6 P l:ol 00L: of ; Arm om. ool ( $=\mathrm{Sh})$, ebenso die Bibelausgaben.

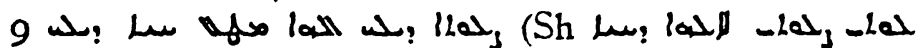

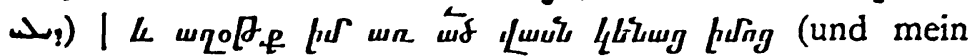
Gebet zu Gott um mein Leben ${ }^{2}$ ).

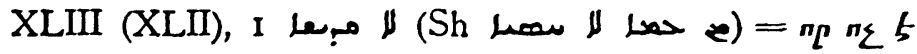
чпце (das nicht heilig ist 3 ).

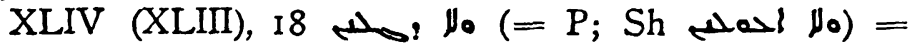

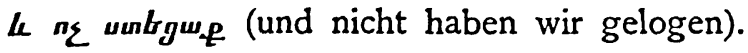

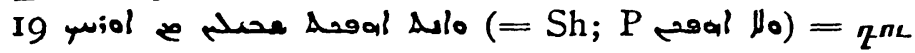

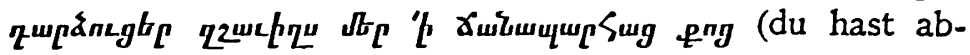
gewendet unsere Pfade von deinen Wegen $\left.{ }^{4}\right)$.

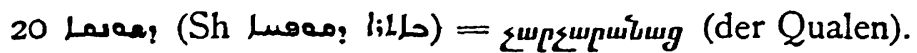

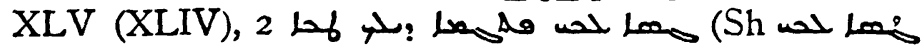
is f wird hervorbringen mein Herz deine guten Worte).

I Es wāre nicht undenkbar, dass Barhebrāus durch das Part. das Praes. (gegen Perf.) bezeichnen wollte. Näher liegt der Schluss, es sei ihm die Stelle bereits syrisch vorgelegen.

2 Eine neue Leseart für die armenische Übersetzung: "mein Gebet, - Gott, um mein Leben."

3 und مبر sind hier synonym.

4 Die kleinen Ab̈weichungen dürfen nicht $z$ weifellos als Varianten gelten, weil es Barhebräus auf "wer" und "du“ ankommt, der Sing. daher leicht der Accommodation an Sh zugeschrieben ist. 


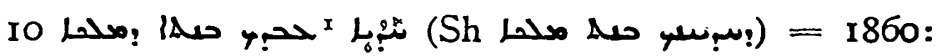

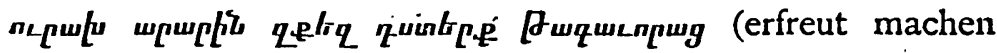
dich die Töchter der Könige ${ }^{2}$ ).

I5 P حمعزحلا Pallis Arm om. (=Sh). - Die armenische Bibel hat statt dessen einen andern Satz. ${ }^{3}$

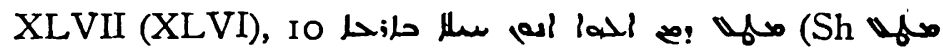
ex;lll $I^{t} \mu_{4} \prod^{5}$ (denn Gottes sind die Mächte auf der Erde ${ }^{4}$ ).

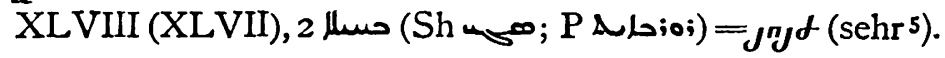

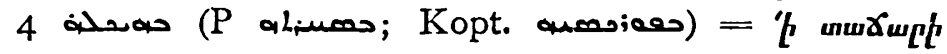
hr [nuL (in seinem oder ihrem Tempel ${ }^{6}$ ).

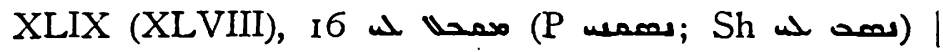

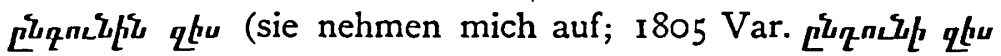
$=$ nimmt er mich auf?).

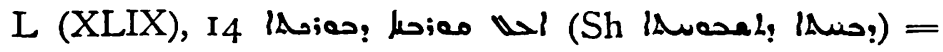

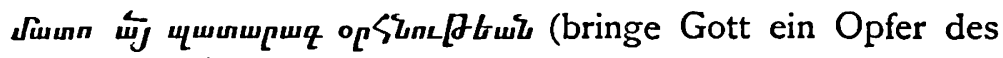
Segens $\left.\operatorname{dar}^{8}\right)$.

1 So Lagarde; S 326 حص? מصن.

2 Die Ausgabe von 1805 trennt den Satz entsprechend der LXX

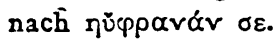

3 Die Stelle ist schon im Hebrāischen verderbt; das Syrische der $P$ bildet das Äquivalent für לרקמות תובל למלך; LXX übersetzt es mit:

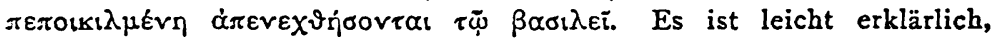
dass von Barhebräus diese Zusammengehörigkeit von $\mathrm{P}$ und Arm nicht erkannt wurde.

4 Barhebrāus setzt hier jedenfalls eine verschiedene armenische Vorlage voraus. (lall e u. (al); jedoch das fehlende Satzende, das im Sh-Citat noch steht, wird er absichtlich ausgelassen haben, weil es nicht in Vergleich gezogen'werden wollte.

5 Das Syrische ist die wörtliche Übersetzung von $\boldsymbol{J}-\boldsymbol{n}^{\boldsymbol{\alpha}}$; es wird adverbiell gebraucht, darum konnte es ebenso mit den andern syrischen Wörtern, von denen es unterschieden wird, gegeben werden; also ist die syrische Fassung des Citates dem Barhebräus wohl schon vorgelegen.

6 Lex. giebt als Bedeutung des armenischen Wortes templum, aedes an.

; Barhebräus kann auch an Sh accommodiert haben, weil es sich für ihn um das richtige Wort blos und nicht um die Form desselben handelt.

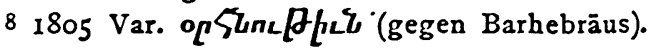




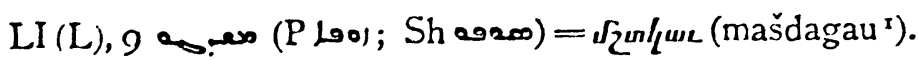

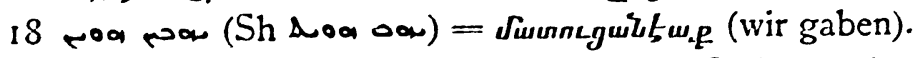

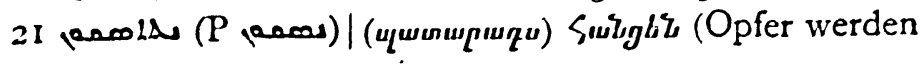
sie darbringen $\left.{ }^{2}\right)$.

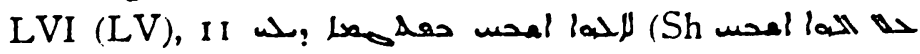

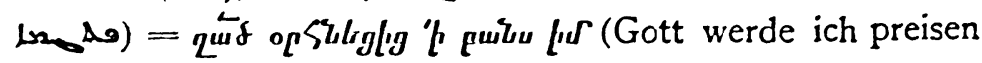
mit meinen Worten).

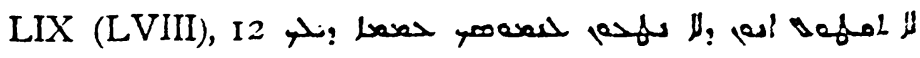

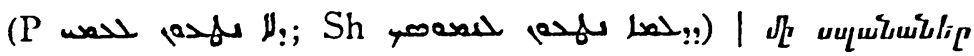

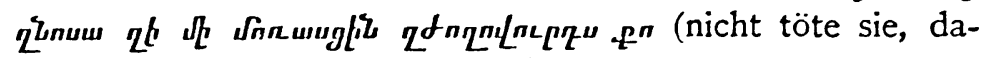
mit sie nicht vergessen deine Völker ${ }^{3}$ ).

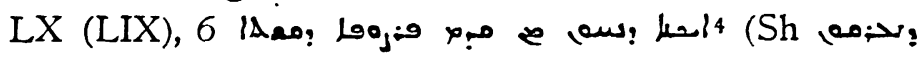
idea : sie gerettet werden vor dem Angesichte des Bogens).

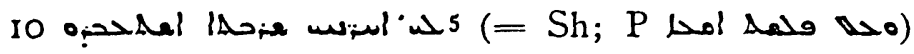

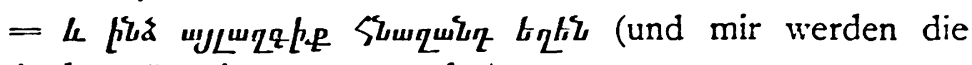
Andersstämmigen unterworfen).

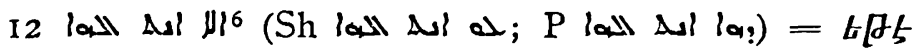
$n_{2} z^{n L}$ iud (wenn nicht du, o Gott).

I Im Zusammenhange zeigt sich, dass hier Barhebräus nicht eine griechische und armenische Bibelstelle anführen will; denn er sagt:

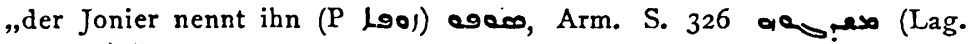
(م). "Und doch sind beide Worte im cas. instrumentalis angeführt. Am besten erklärt sich die Sachlage, wenn beide Worte am Rande einer Hs. gestanden haben und von Barhebrāus irrtümlich als Nom. gefasst worden sind; vergl. übrigens p. 126 Anm. 2.

2 Es ist kein Zweifel, dass Arm den Gedanken aktiv ausdrūckt;

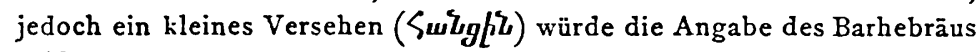
erklären.

3 Nach Barhebräus böte der Armenier die Lesearten von $P$ und Sh vereint; jedoch die Ausgaben haben nichts davon. - Die verschiedenen Übersetzungen sind Versuche, die schwierige hebräische Stelle verständlich zu machen.

4 S 326 hat poud ind 00 jo.

5 S 326 hat

6 S 326 lässt lall aus. 


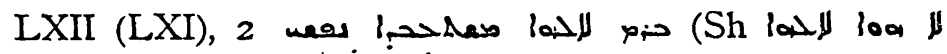

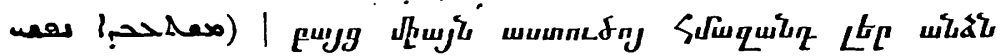
Lif (jedoch allein Gott ist meine Seele unterworfen).

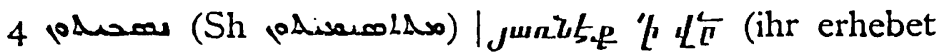
euch gegen $\left.\ldots{ }^{\mathrm{x}}\right)$.

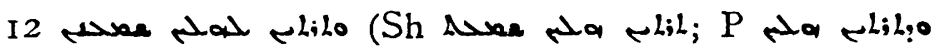

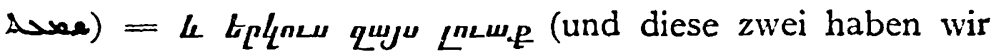
gehört $\left.{ }^{2}\right)$.

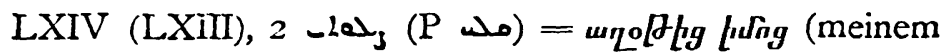
Gebete).

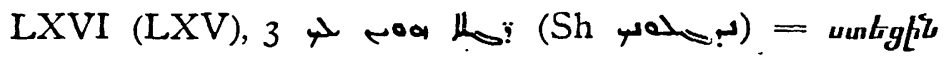
$f^{t} \mathbf{q}$ (sie logen dir $\left.{ }^{3}\right)$.

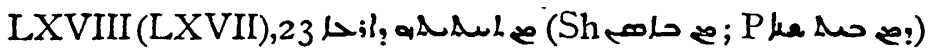
"1 luning wingluqng (von den Abgründen der Tiefen 4).

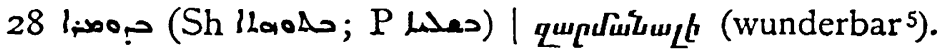

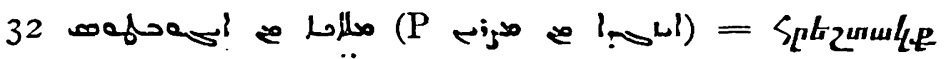
ftaqlumanub (Boten aus Ägypten).

I Es liegt ein Versehen des Barhebräus oder seines Gewährsmannes

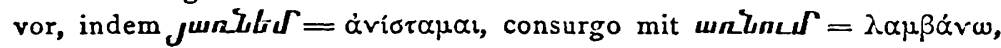
capio, sumo verwechselt wurde.

2 Die Stellung der nota acc: \& zum 2. Worte erklärlich aus dem Armenischen, wo $q$ ebenfalls erst beim 2. Worte steht. Das Syrische könnte man auch übersetzen: „und zweitens haben wir dieses gehört.“

3 Inhaltlich besagen beide Citate das Gleiche; der Unterschied zwischen Arm und Sh ist nur ein formeller.

- 4 Eigentlich würde der 2. Versteil des armenischen Textes dem 1. Teile der Sh entsprechen; die Umstellung des armenischen Textes ist auch Barhebrāus schon vorgelegen. - Die Leseart des Barhebrāus Lحil? dürfte einem der vielen Versehen desselben zuzuschreiben sein: wilqng

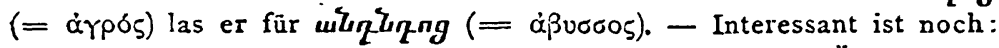
für حامي (Bacav) bietet der armenische Text die gleiche Übersetzung wie P: "von zwischen den Zähnen“ gegen LXX; vergl. ob. zu XVI, 2.

5 Es ist wahrscheinlich, dass die Differenz auf einer Accommodation an die anderen Citate zurückzuführen ist. 


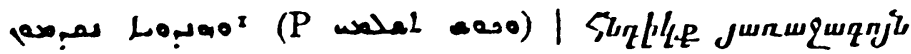
abniunne Liglis (die Indier werden vorher Helfer sein ${ }^{2}$ ).

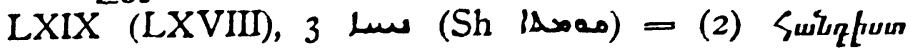
(Ruhe 3).

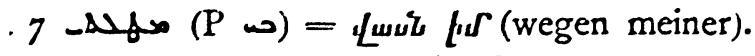

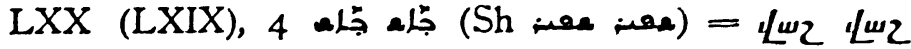
(ach, ach 4).

LXXII (LXXI), I ب L ف (

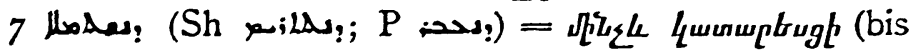
dass er vollendet wird).

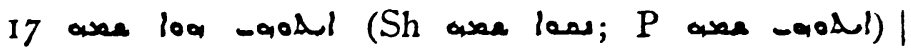

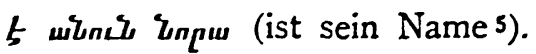

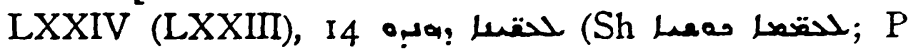

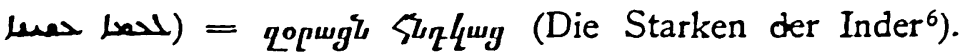

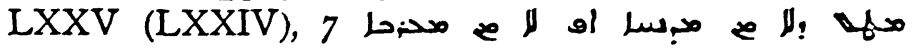

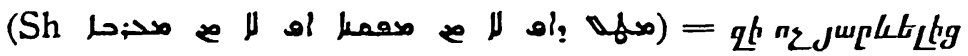

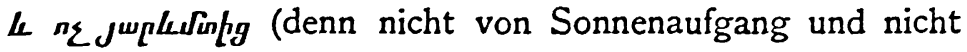
von Sonnenuntergang).

I Lagarde zieht auch noch das nachfolgende ! l zum armenischen Citat; jedoch mit Unrecht; denn das Citat des Arm selbst giebt blos die Umschreibung für rpo-; das Folgende ist anders gefasst; gehört wieder zu P.

2 Das Citat zeigt, dass nur das adv. junuquqnj mit Lkght herausgerissen und zusammengenommen wurde ohne Rücksicht auf den Zusammenhang.

3 Die Vorlage des armenischen Übersetzers scheint $\dot{\alpha} \tau 0 \sigma r \alpha \sigma ı 5$ gewesen zu sein (so $B^{a}$ bei Swete).

4 Lagarde bemerkt hierzu: Armeni waš non baš habent; jedoch weiches $\Delta$ und $\mathcal{L}$ entsprechen sich auch sonst im Armenischen und Syrischen cf. Hübschmann, Armenische Gramm. I. p. 286. - Die eigentūmliche Punktation (ج) zeigt, dass Barhebrāus das Wort als syrische Transscription erkannte.

5 Barhebrãus erklārt Sh für richtig, Arm für nicht richtig. Es kommt ihm hier offenbar auf das Tempus an: Fut. u. Perf. Nach Barhebräus hat Arm. das Perfekt: ,ist gewesen“.

6 Auch hier ist wieder eine aus $P$ und IXX combinierte Leseart beim Armenier zu finden; vergl. LIX, 12. 


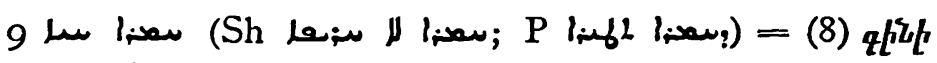
Li

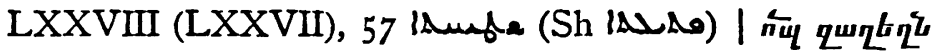
Pl $\mathbf{L}_{\mathbf{L}}$ (wie ein verkrümmter Bogen $\left.=\mathrm{Sh}^{2}\right)$.

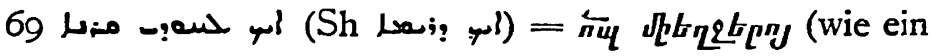
Einhorn).

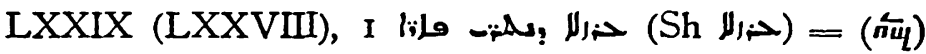

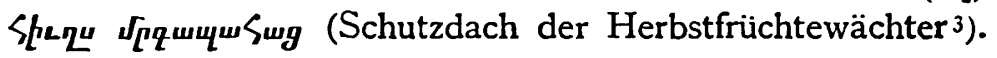

LXXX (LXXIX), 9 مفل Linfutgitp (du hast geändert).

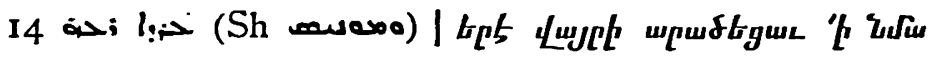
(das Tier des Feldes weidete darauf 4 ).

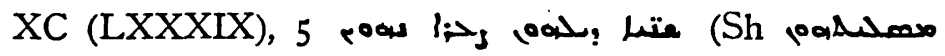

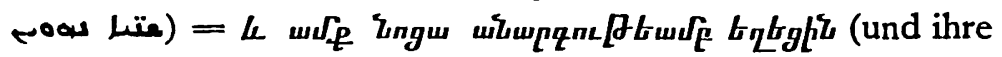
Jahre werden zur Schande sein 5 ).

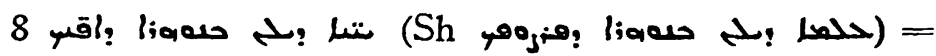

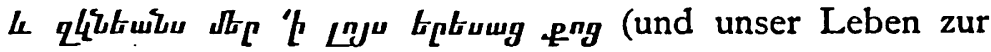
Leuchte deines Angesichtes).

I wird für purus, merus vom Weine gebraucht; vergl. P. S.

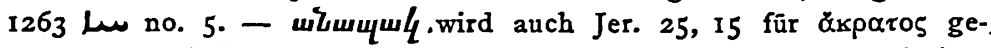
braucht; wörtlich übersetzt hiesse es : « $\varphi \vartheta \alpha p r o 5$, was man in der syrischen Wiedergabe auch ausgedrückt $z$ u finden erwartete.

2 Barhebräus (,ebener Bogen“) scheint sich versehen zu haben

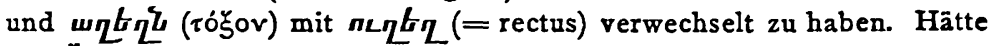
der Übersetzer ins Syriścine; die ganze Stelle übersetzt, so wäre eine Verwechslung dieser Art nicht vorgekommen.

3 Ob Barhebrãus eiร oder ís las, ist nicht zu entscheiden.

4 Es fallt auf, dass hier nicht wie sonst das Armenische wörtlich wiedergegeben ist. Bemerkenswert ist, dass die syrohexaplarische Hs., die Ceriani verōffentlicht hat, $l_{9}$ als Randleseart bietet ohne Angabe der Herkunft.

5 Die kleine Variante ("Schande“ und „zur Schande") mag der Accommodation an $\mathrm{Sh}$ entsprungen sein. 


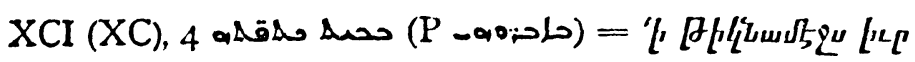
(mit seinen Schultern ${ }^{\text {) }}$ ).

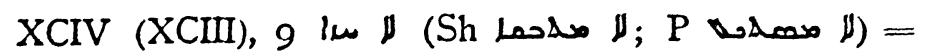
nc untrumilgyt (nicht sollte er sehen ${ }^{2}$ ).

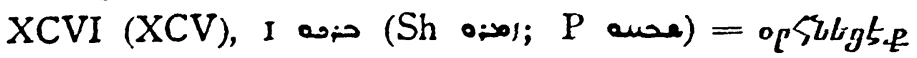
(preiset).

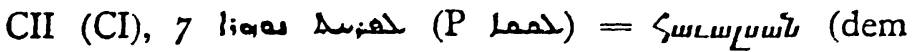
Pelikane 3 ).

CIV (CIII), I u. 2 منده (d. i. I. sg.

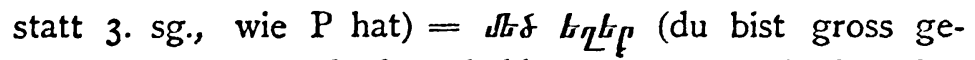
worden), qqtgup (du hast bekleidet), wүLwp (du hast bedeckt), aqtght (du hast gespannt).

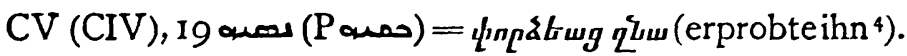

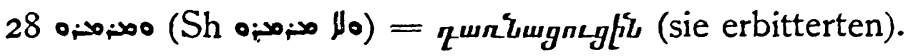

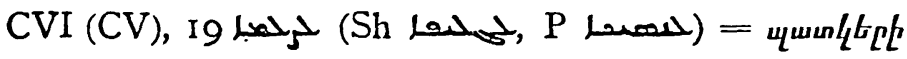
(Bild s).

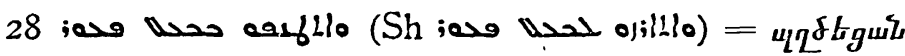

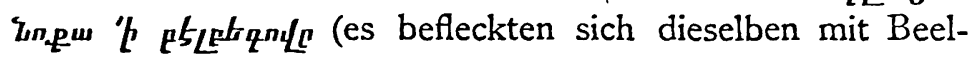
phegor).

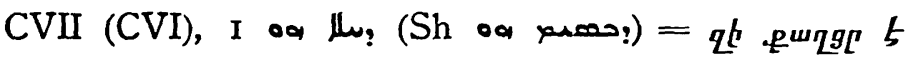
(denn süss ist $\mathrm{er}^{6}$ ).

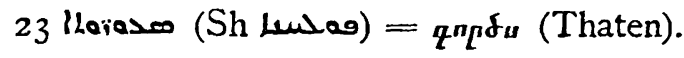

I Das Syrische kennzeichnet sich als wörtliche Wiedergabe des Armenischen ("mit dem 2 wischen den Schultern"). treten sein.

2 Das armenische Wort kann durch alle 3 syrischen Verba ver-

3 Das armenische Wort ist ein Compositum aus SuL $=$ avis und $\left\langle\boldsymbol{J}^{\boldsymbol{u}}=\operatorname{lux}\right.$ (= syr : „Feuervogel").

4 Arm u. P haben synonyme Worte.

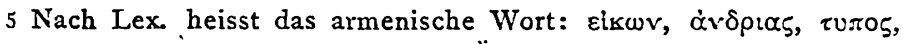
$\dot{\alpha} \gamma \alpha \lambda \mu \alpha$, also könnten alle 3 syrischen Übersetzungen dafür genommen werden.

6 Loftusius in „Excerpta“ bei Walton VI irrtümlich : وسللم quia fortis“،. 


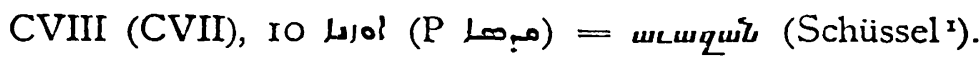

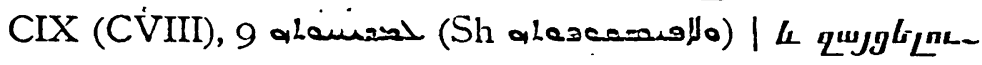
BfpL Lnn (und seine Beaufsichtigung ${ }^{2}$ ).

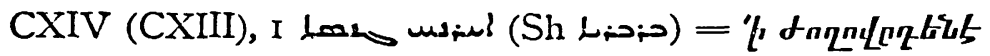

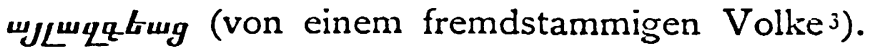

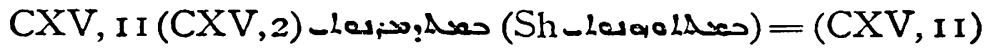

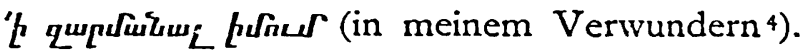

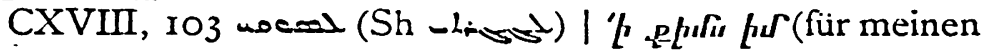
Gaumen 5).

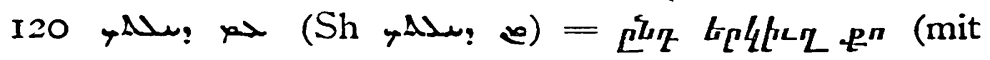
deiner Furcht).

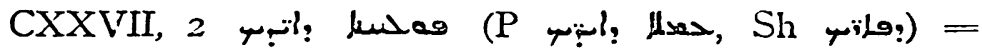
qLumunuLu átrumg png (die Arbeiten deiner Hände ${ }^{6}$ ).

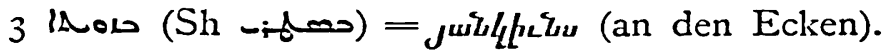

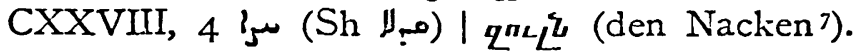

I Beide Worte, das syrische und armenische, sind verwandt; nach Hübschmann, Arm. Gr. I. p. III stammen sie aus dem Persischen.

2 Auch hier dürfte die Erklärung der Leseart in einer Verwechslung liegen: Barh. oder sein Gewährsmann lasen fälschlich oft_nL $\partial \not h L$ $=$ ofnL $f_{f i}\{=x p i \sigma \mu \alpha$, unctio (nach Lex.).

3 Das Syrische ist die wortwörtliche Viedergabe des armenischen Compositums ( $\boldsymbol{\omega} \mathcal{L}=$ alius, $\boldsymbol{w g} \underline{\mathrm{g}}=$ genus).

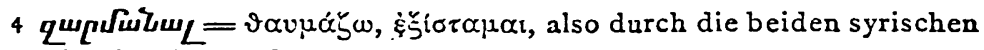
Worte richtig übersetzbar.

5 Barhebrāus ("für meinen Geruch") scheint eine verschiedene Leseart zu bieten; möglicherweise kann auch eine Verwechslung des Sinnes vorliegen bei dem Übersetzer aus dem Armenischen. - Eine blosse Vermutung, wenn aach der armenischen Übersetzung sowie den oftmaligen Missgriffen des Barhebräus entsprechend, wäre die Annahme, die armenische Übersetzung haje hier verbessert und statt $\lambda \propto \rho u \gamma \gamma^{2}$ gesetzt Snql ( $\pi v \varepsilon v j \alpha)$; dieses mit Snu (odor) zu verwechseln, wäre unschwer anzunehmen.

6 حملا u. vönnten in gleicher Weise das armenische Wort wiedergeben.

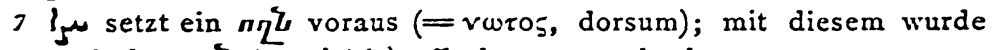
das armenische mL $\mathcal{L}$ cijiniv) offenbar verwechselt. 


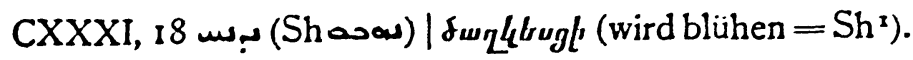

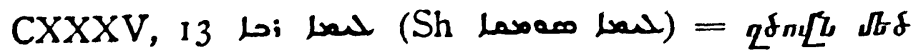
(das grosse Meer).

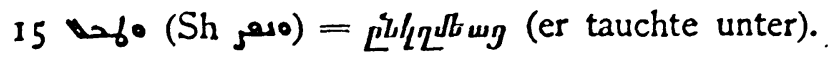

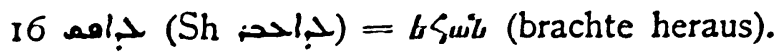

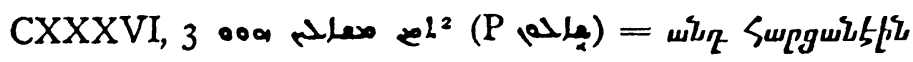
(dort fragten sie 3 ).

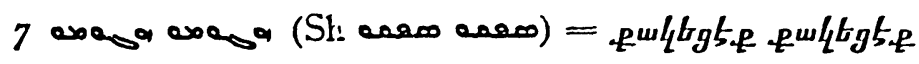
(zerstöret, zerstöret4).

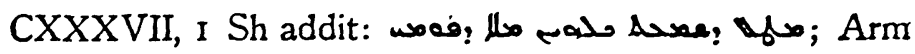
om. $=$ P. Jedoch thatsächlich hat der Armenier diesen Versteil am Schlusse des Verses5.

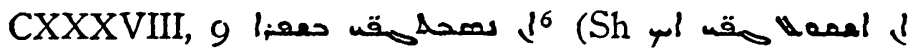

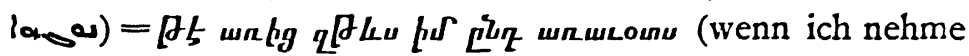
meine Flügel am Morgen 7 ). hindern).

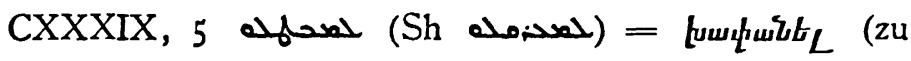

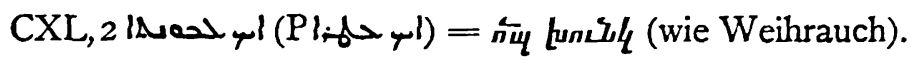

I $\delta_{u q} t \omega=\alpha \dot{ } \boldsymbol{\alpha} \alpha \tau \lambda \lambda \lambda \omega=\omega$; offenbar wurden beide ähnlichen IVorte verwechselt.

2 el lässt Lagarde aus, S, 326 setzt es.

3 Barhebräus punktiert das Citat aus $P$ und fügt hinzu: ,von لles und nicht von IN/a." Offenbar soll damit das $\mathrm{Pa}$ festgestellt werden

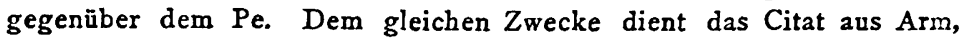
bei welchem die Participform sofort für das $\mathrm{Pa}$ entscheidet - ein neuer Beleg, dass Arm dem Barhebräus bereits syrisch vorlag.

4 Sh und Arm haben synonyme Worte.

5 Die griechischen Hsn. haben den Versteil z. T. da, wo ihn Sh des Barhebräus voraussetzt, Hs. A bei Swete hat ihn da nicht. Am Schlusse des Verses bieten ihn einzelne Hsn. Die Angabe des Barhebrãus stimmt also mit dem genauen Sachverhalt.

6 In Eichhorn, Repertorium XIII p. 196 wird \ ausgelassen. liae ist.

7 Die koptische Übersetzung bietet حرف: was nur ein Synonym zu 


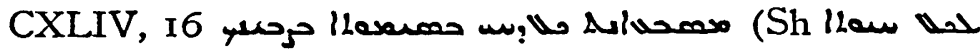

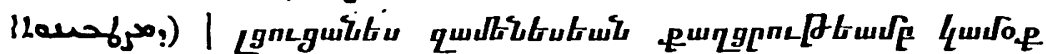
fruLe (du erfülltest alles mit Süssigkeiten nach deinem Willen ${ }^{\mathrm{x}}$.

Angesichts dieser zahlreichen (I59) Citate unter der

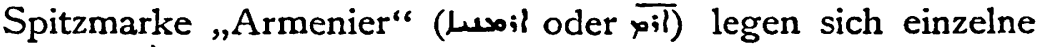
Fragen nahe. Dass die Citate wirklich der armenischen Bibelübersetzung entnommen sind, haben wir in den Vorbemerkungen vorausgesetzt und finden es jetzt vollauf bestätigt. Die grösste Anzahl der Stellen stimmt ja mit der genannten Übersetzung vollkommen überein; zudem kennzeichnen sich viele als wörtliche Übertragung der armenischen Vorlage ${ }^{2}$; was aber vor allem die Thatsache ausser Zweifel stellt, sind die Stellen, wo Missverständnisse des armenischen Textes vorliegen ${ }^{3}$, und an denen der armenische Text unübersetzt herübergenommen wurde 4.

Damit ist aber noch nicht entschieden, ob Barhebräus selbst die armenische Übersetzung beigezogen hat oder ob ihm die angeführten Stellen schon syrisch vorlagen. Dass er armenisch verstand, lässt sich voraussetzen wegen der nahen Berührung mit den Armeniern. . Positive Beweise für diese Kenntnisse dürfen wir wohl in der Transscription von armenischen Worten finden; denn ohne Verständnis wird er diese nicht angeführt hahen4. Die genaue Bezeichnung

I Statt „alles" hat Barhebräus: „alles Lebende“ (= LXX u. P); möglicherweise ist dies aus $P$ einfach herübergenommen; jedoch kann auch eine eigene Leseart darin bezeugt sein, falls nicht Barhebrāus sich versehen und qualtitutuit in ,alles" und "Leben" (4tuitu) zertrennt hat.

2 Vergl. 1,$4 ; 7,18 ; 18,50 ; 27,11 ; 29,10 ; 45,10 ; 48,2 ; 60,10$; 62,$12 ; 91,4 ; 102,7 ; 114,1$.

3 Vergl. zu Lev. 11, 19; Ps. 17, 14; 21, 4; 35, 21;62, 4; 78, 57 ; 109,$9 ; 128,4 ; 131,18$.

4 Vergl. Lev. 11, 19; Ps. 5, 10; 51, 9; 70, 4 . 
der Aussprache zu Ps. 70, 4 lïsst das Gleiche erschliessen. Vor allem aber verrat er ein Verständnis der armenischen Spraclic, wenn er l's. 22, 28 benerkt, dass der Armenier lautlich das gleiche Wort setze wie im Syrischen (vol. zur Stelle). Jedoch wenn dem Barhebräus keineswergs das nötige Verstindnis fehlte, um den armenischen Bibeltext in Einsicht $\% u$ nehmen, so weisen andererseits vicle Erscheinungen darauf hin, dass ihm die Citate aus der armenischen Ubersetzung bereits in syrischer Sprache vorlaren. Wiscman" findet ein Hauptargrument dafür in den mannigfachen Punktationen, dic Barhebräus zu armenischen Citaten anfülurt z. 13 . 1's. 5, 10; 8, 5. „Wic könnte eine solche Erörterungr über die Punktation statthaben, wenn es sich um den armenischen Text handelte?" Bei genaucrer Beobachtung wird dieses Argument hinfällig. Wollte Barhebräus seinen Landsleuten dic armenische Lesart z. B. I's. 8, 5 verständlich machen, so konnte das nur durch Angabe der verschicdenen Punktation bei gleichlautendem Consonantenbestande geschehen. Was vollends Lie Ps. 5, 10 betrifft, so ist darin durchaus keine syrische l'unktation zu schen, sondern die Punktation dient nur der lautlichen Wiedergabe der armenischen Volialc2. Ebenso ist in Ps. 70, 4 durchaus kine syrische Punktation zu schen; was sollte es bedeuten im Syrischen ein a im Anlaut mit $R$ zu punkticren? 13arhebräus will viclmehr die Aussprache des Armenischen' in der Umschrcibung möglichst genau angeben. Ist also in der Begriindung Wiscman nicht zu folgen, so bestcht trotzdem scine Anschauung zu recht, dass die Citate dem Barhebräus zum grössten Teile nicht selbst armenisch vorgelegen.

I Ilorae syriacae p. 143.

2 Wiseman wurcle durch das irrtümlich dazugyckommene Suffix (1. sg.) verleitet, darin ein syrisches Wort mit eigentümlicher Vokalisierung zu erblicken. 
Sehr oft ist nämlich der Fall gegeben, dass die armenische Vorlage durch verschiedene syrische Wörter gegeben werden kann. Lag nun eine dieser möglichen Übersetzungen schon vor im P- oder Sh-Citate, so versäumt es Barhebräus trotzdem nicht, auch die armenische Übersetzung noch eigens in abweichender syrischer Fassung wiederzugeben. Manchmal mag ja der Sprachgebrauch die eine von verschiedenen Auffassungen überwiegend erscheinen lassen ${ }^{x}$; bei den meisten dergleichen Differenzen ist auch dies nicht möglich, und in diesem Falle kann der Exeget, der den armenischen Text selbst einsieht, eine Variante selbstverständlich nicht darin finden und nicht notieren. ${ }^{2}$ Mehr noch gilt dies, wenn es sich um gleichbedeutende Formen desselben Wortes handelt z. B. I, 6; 27, I; 4I, 4; 66, 3; 136, 3 . Besonders die letzte Stelle scheint markant zu sein. Barhebräus will hier feststellen, dass das $\mathrm{Pa}$ von whe (statt des $\mathrm{Pe}$ ) stehe; er besorgt dies durch Punktation und durch Hinweis auf die armenische Leseart, deren participiale Ausdrucksweise $\mathrm{Pa}$ unzweifelhaft macht. Das kann aber nur gèschehen, wenn der Text dem Barhebräus schon syrisch vorgelegen. Ebenso erklärt sich am besten die Bemerkung zu Ps. 22, 28, wenn bereits syrisch die Übersetzung lsö̈ vorlag als Synonym zu dem ids; der P.

Wenn nun die Citate aus der armenischen Übersetzung dem Barhebräus bereits syrisch vorlagen, sind sie einer vollständigen syrischen Übersetzung aus dem Armenischen entnommen, und woher stammen denn die Transscriptionen ins Syrische? Wiseman fühlte sich versucht zu sagen, die Syrer hätten eine einheimische Übersetzung, aus der arme-

I Vergl. zu Ps. I, I.

2 Wir haben jeweils in den Anmerkungen darauf hingewiesen, unterlassen es darum diese Stellen noch ausdrücklich nambaft zu machen. 
nischen gefertigt, besessen. ${ }^{*}$ Dagegen spricht jedoch manches. Einmal müsste dann eine doppelte Quelle der syro-armenischen Citate des Barhebräus angenommen werden : die supponierte syrische Übersetzung und der armenische Text selbst; denn die Transscriptionen aus dem Armenischen entstammen dem Bibeltext, sind nicht blos Spuren von der Kenntnis des Armenischen. Weiterhin sind manche Versehen derart, dass sie nur bei einem raschen Vergleich, beschränkt auf das in Betracht $z u$ ziehende Wort, sich erklären lassen; so Lev. 11, 19, wo die Zeilenrichtung des Syrischen auf das Armenische übertragen wurde; Ps. 78, 57 wurden Substantiv und Adjectiv verwechselt. ${ }^{2}$ Überhaupt ist die verhältnismässig grosse Anzahl von Versehen eher möglich bei stellenweisem Vergleiche und auf diese Stellen beschränkter Übersetzung, als bei einer zusammenhängenden Übertragung. So möchte denn am ehesten allen Eigentümlichkeiten der vorliegenden Citate und zugleich der allgemeinen Anlage der Scholien des Barhebräus die Anschauung gerecht werden: die syro-armenischen Citate hat Barhebräus als Randnoten zu seinen Handschriften vorgefunden, wie auch andere, namentlich syro-hexaplarische Citate. Dadurch erklärt sich auch am besten die Mischung von syrischen Übersetzungen mit blossen Transscriptionen, die ein Analogon in den auch züm Teil in griechischer Sprache sich vorfindenden Randnoten der syrohexaplarischen Handschriften haben. Daraus und nur daraus erklärt sich auch ein eigentümliches Citat, Ps. 51, 9; zu der syrischen Beschreibung des. Hysop fügt Barhebräus hinzu: „der Grieche nennt ihn 0 , der Armenier ob:e. $3^{\text {"s }}$ Ersteres kann nichts anderes sein als die Transscription einer griechischen Randnote ن்бол $\omega$ im

I Hor. syr. p. 143.

2 Vergl. auch zu LXVIII, 32.

3 Wenn Lagarde richtig korrigiert. S 326 ist undeutlich; Hs. Sachau 134 hat, wie ich eben sehe, blos 
Dativ, letzteres steht in dem entsprechenden Instrumentalis; Barhebräus aber umschreibt beide und verwendet sie, als ob die Worte im Nominativ angeführt wären. Aus dem Context genommen, könnten sie nicht so gebraucht werden; denn da hätte Barhebräus nicht übersehen können, dass die Worte nicht die absolute Form haben, sondern in abhängiger Form angeführt sind.

So haben wir in den syro-armenischen Citaten des Barhebräus zwar nicht Überreste einer syrischen Bibelübersetzung, wohl aber Überbleibsel einer armenischen Handschrift in syrischer Übertragung vor uns. Es frägt sich weiter, ob diese Citate auch einen Ertrag abwerfen für die Erforschung der armenischen Bibelübersetzung und weiterhin für deren griechische Quelle. Wenn wir von der interessanten allgemeinen Bemerkung des Barhebräus zu Ps. 16, 2 absehen, so ist wohl einiger, wenn auch geringer Ertrag für die armenische Textkritik aus diesen Citaten zu entnehmen. Selbständige Lesearten sind wenige $\mathrm{zu}$ finden, aber es sind solche da, und sie sind zur Textkritik zu verwerten. ${ }^{x}$ Zugleich sird diejenigen Stellen nicht ohne Bedeutung, welche teils für einzelne Varianten sich entscheiden, ${ }^{2}$ teils die gemeinsame Leseart der armenischen Handschriften bestätigen. 3 Weiter noch der Bedeutung der einzelnen Varianten nachzugehen, ist von keinem Nutzen, weil die Textkritik der armenischen Übersetzung noch ziemlich im argen liegt 4 und die verhältnismässig geringe $Z \mathrm{ahl}$ der in Betracht zu ziehenden Citate keine sicheren' Schlüsse ergeben würde.

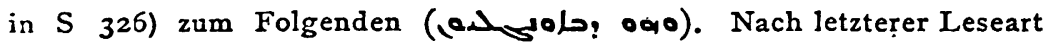
würde diese Stelle nichts beweisen.

I Ps. $18,8.26 ;(28,8) ; 30$, 10; 36,$7 ; 39,12 ; 40,7 ; 41,10 ; 42,9$; 47,$10 ;(51,21) ; 59,12 ; 62,2 ; 72,17 ;(118,103 ; 144,16)$.

2 Ps. 16,$2 ; 22,10 ; 45,10 ; 49,16$.

3 Diese Stellen sind die übergrosse Mehrzahl.

4 Vergl. Vigouroux, Dictionnaire de la Bible I. 1014. 


\section{Die syro-koptischen Bibelcitate.}

Nicht so leicht, wie dic Beiziehung der armenischen Bibelübersetzung erlklärt es sich, warum der Schrifterklärer aus Grossarmenien nach einer Übersetzung des fernen $\ddot{A}$ gypten griff, um seine ostsyrischen Schüler in die Textkritik der hl. Schrift einzuführen. Bei näherer Erwägung jedoch stellt sich diese Thatsache keineswegs als vereinzelt dar. Dass eine Verbindung zwischen den monophysitischen Kopten und den jakobitischen Syrern stattgehabt habe, lässt die Verwandtschaft ihrer dogmatischen Anschauungen erwarten. Auch finden wir in der Geschichte erwähnt, dass einmal ein Kopte berufen war, zwischen Jakobiten und Moslim zu vermitteln. ${ }^{x}$ Einen Beweis für literarischen Austausch, ja sogar für bibelkritische Verwertung der koptischen Übersetzung finden wir in einer polyglotten Psalterhandschrift, die neben dem syrischen und äthiopischen, armenischen und arabischen Texte auch den koptischen enthält. ${ }^{2}$ Also war für Barhebräus die Möglichkeit gegeben und bei seinen Schülern und Lesern das nötige Interesse vorhanden, um unter die textkritischen Hilfsmittel auch die koptische Übersetzung einzureihen.

Barhebräus citiert diese Übersetzung unter dem Namen

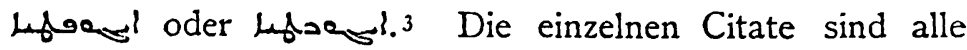
dem Buche der Psalmen entnommen. Nur in der Einleitung zu den Proverbia wird im allgemeinen auf die koptische Übersetzung hingewiesen: „die Griechen und die Kopten,“

I Vergl. Nöldeke, Orientalische Skizzen p. 258.

2 Vergl. Wiseman, 1. c. p. I44 s, Revue bibl. 1896 p. 542 (cod. Barb. 3 1).

3 Wiseman 1. c. p. 143 und nach ihm Rhode 1. c. p. 19s haben mit Recht darunter die koptische Bibelübersetzung verstanden; nach P. S. p. 137 erklärt Barhebräus im Chronicon selbst dieses Wort mit: ,die Orthodoxen in Ägypten"; in BB p. 23 1. 10 ist ofoes' mit قُ erklärt. 
sagt dort Barhebräus, $\mathbf{x}$,haben den grössten Teil der Salomonischen Sprüche verloren"."

Auch die koptischen Bibelcitate sind nur gelegentlich bisher berührt worden. 3 Im Folgenden soll eine erschöpfende Zusammenstellung derselben geboten und ein Vergleich mit den vorhandenen koptischen Texten versucht werden in ähnlicher Anlage wie bei den syro-armenischen Citaten. Der zu Grunde gelegte Scholientext des Barhebräus ist der gleiche wie bei den syro-armenischen Citaten; 4 der koptische Text ist der Ausgabe von Ciasca entnommen für die sahidische Übersetzung, die memphitische Übersetzung wurde nach Lagarde's Ausgabe beigezogen. 5 Daran soll sich eine Erörterung über Wert und Bedeutung dieser syro-koptischen Bibelcitate des Barhebräus schliessen, soweit es der Zustand des koptischen Bibeltextes zulässt.

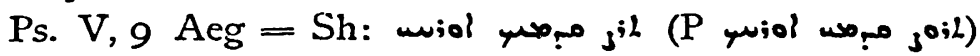

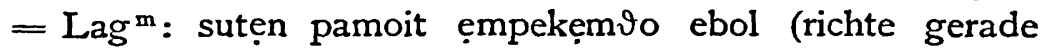
meinen Weg in deiner Gegenwart); dagegen Pey (v. 8): COOYTN NTEKZIH MாAMTO EBOA (richte gerade deinen Weg in meiner Gegenwart $=$ P). ${ }^{6}$

I Vergl. Rahlfs, des Gregorius Abulfarag, genannt Bar Ebroio, Anmerkungen zu den Salomonischen Schriften (Leipzig, 1887) p. 2.

2 Vergl. Ciasca, Fragmenta copto-sahidica II. p. XL-XLVI, wo wenigstens für die sahidische Bibelübersetzung verschiedene Auslassungen constatiert werden. - Mit dieser Beschränkung ist die Notiz bei Rhode, 1. c. p. 19 und Wiseman 1. c. p. $142 \mathrm{zu}$ versehen, dass die koptische Übersetzung nur zu den Psalmen citiert werde.

3 So von Bruns in Eichhorn, Repertorium XIII p. 186, Wiseman, hor. syr. p. 144, Rhode 1. c. p. 19 u. 74.

4 Vergl. oben p. 102.

5 Ciasca A., Sacrorum Bibliorum Fragmenta Copto-Sahidica, Rom 1889, II; die Lücken sind in den Anmerkungen ergänzt aus Peyron B., Psalterii Copto-Thebani specimen, Aug. Taur. 1875 und Lagarde, Psalterii versio memphitica: Accedunt Psalterii Thebani fragmenta, Berlin 1875. Abkürzungen: Ci, Pey, Lagth, Lagm.

6 In $\mathrm{Ci}$ ist hier eine Lücke; auch die Fragmente des thebanischen Zeitschrift f. d. alttest. Wiss. Jahrg. 2r. I. Ig0x. 


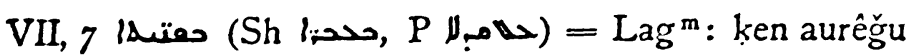
- Pey (v. 6): 2П Пटан (an den Enden, Grenzen). ${ }^{\text {ॠ }}$

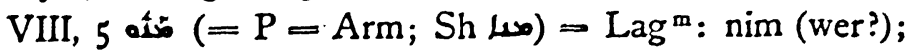
$\mathrm{Ci}$ : or (was?). ${ }^{2}$

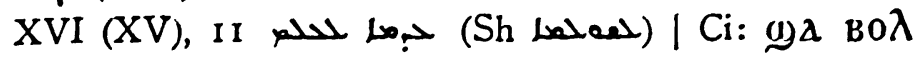

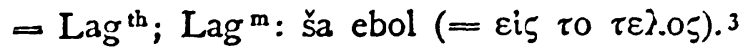

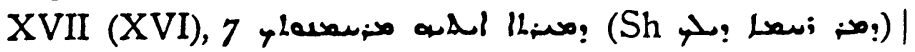

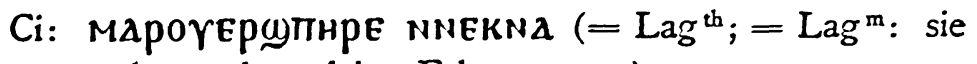
mögen bewundern deine Erbarmungen). 4

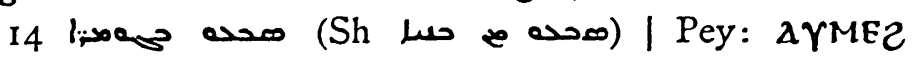
2HTOY NAY NOUE (= Lag ${ }^{\text {th }}$ : sie füllten ihr Inneres mit dem Fleisch der Söhne); Lag $^{\mathrm{m}}$ : ẹnaf ẹnrir (= mit dem Fleische der Schweine). 5

(= sahidischen) Psalteriums bei Lagarde sind am Anfang verstümmelt. Lagm bietet den memphitischen Text nur in Umschrift. - Auch der LXX Text bietet nach Swete abweichende Lesearten, wenn auch die genaue Vorlage der Leseart des Barhebräus nicht vorhanden ist.

I Aeg und $\mathrm{Sh}$ bieten Synonyma.

2 Barhebräus und Lagm bieten die Leseart des Cod. Alex. (= A nach Swete).

3 Barhebräus setzt die Leseart $\boldsymbol{\omega} \mathbf{\lambda}$ ZENEZ (bis in Ewigkeit) voraus. Eigentümlich ist, dass die erwartete Vorlage gerade am Schlusse des vorhergehenden Psalmes sich findet. Eine Einsichtnahme des koptischen Textes von Fall zu Fall, wie wir es für die armenische Übersetzung annehmen zu sollen glaubten, lässt ein Abirren des Auges vom Schluss des XVI. Psalmes zum Schluss des vorhergehenden Psalmes bei An. klang der Form $(\omega \boldsymbol{\Lambda})$ und Verwandtschaft der Bedeutung sehr gut als möglich erscheinen. - Die äthiopische Übersetzung hat übrigens

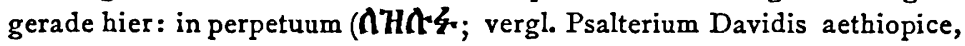
London 1815).

4 Nach dem koptischen Satzbau ist die Auffassung des Barhebrāus wohl zu erklären: $\bar{p}$ WTнрЕ heisst auch mirabilis, $\bar{p}=$ esse, also: es mögen bewundert sein; das Akkusativzeichen $\bar{N}$ vor NEKNג war leicht zu übersehen.

5 In den beiden, koptischen Übersetzungen sind die Lesearten $\dot{v} \varepsilon \imath \omega r$ und $\dot{\omega} \omega r$ vertreten. $\omega_{\mathrm{E}} \mathrm{E}$ ist eine Abkürzung für $\omega_{\mathrm{H}} \mathrm{pE}$; zugleich heisst es aber auch "lignum" Holz. Es ist leicht begreiflich, dass aus dem „Fleisch des Holzes" „Kohlen“ zurecht gemacht wurden im Hin- 
I | Pey:

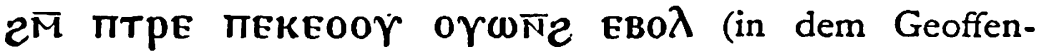

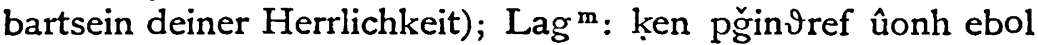
ẹğe pekôu (in ihrem sich Offenbaren, nämlich deiner Herrlichkeit).

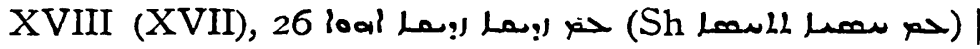

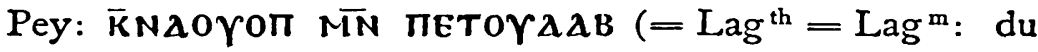
wirst heilig sein mit dem Heiligen $=$ Sh gegen Barh.: ich werde sein).

Sh loal

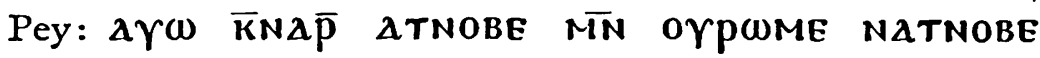
$\left(=\mathrm{Lag}^{\text {th }}=\mathrm{Lag}^{\mathrm{m}}\right.$ : und $\mathrm{du}$ wirst schuldlos sein mit dem schuldlosen Manne).

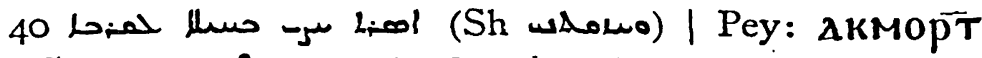

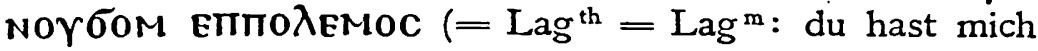
umgürtet mit Kraft zum Kriege.) ${ }^{x}$

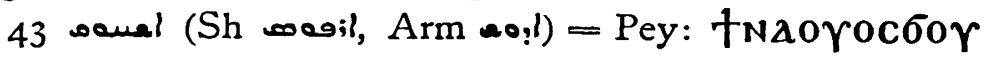
(= Lag $^{\text {th }} ; \operatorname{Lag}^{\mathrm{m}}$ eiefotu ich werde sie zertreten.) ${ }^{2}$

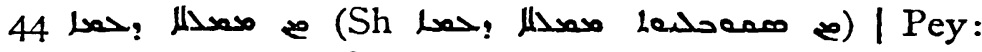

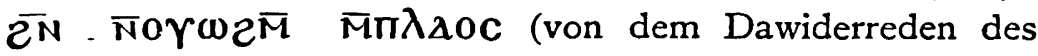
Volkes $=$ Lag $^{\text {th }} ;$ Lag $^{m}$ hat antilogia. $)^{3}$

blick auf die falsche Lesung beim Armenier; also: „sie sättigten sich mit Koblen." - Bruns meinte in Rep. XIII p. 186, der Ägypter und Armenier hätten das ص 2 der Peschittho in lias abgeändert; nach Obigem ist dem nicht so.

I Es ist nicht ganz sicher, dass Barhebräus hier den Kopten ganz genau citieren wollte; vieileicht kam es ihm nur auf den Unterschied „bekleiden" und „umgürten“, „im Kriege“ (= Arm.) und „zum Kriege“ an, während das Übrige dem Armenischen accommodiert wurde.

2 Die syrischen Varianten scheinen nur synonyme Ausdrücke für den gleichen allgemeinen Sinn; auch die thebanische und memphitische Übersetzung kommen in der allgemeinen Bedeutung ,vernichten“ überein.

3 Hier stimmt Barhebrāus mehr mit der thebanischen Leseart überein, weil ein Wort den Begriff giebt; die memphitische Leseart stimmt zu Sh. 


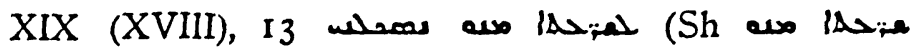

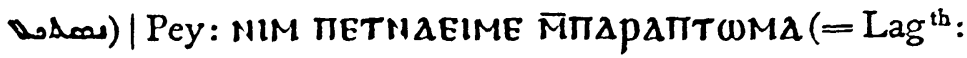

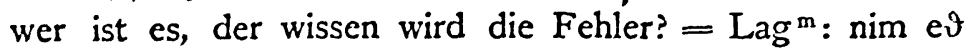
naškati ehanparaptôma.) ${ }^{x}$

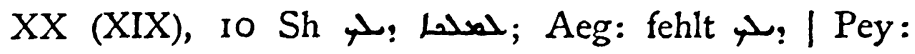
пекр̄po (deinen König $=$ Lag $^{\text {th }}=\operatorname{Lag}^{\mathrm{m}}$ : ẹmpekuro).

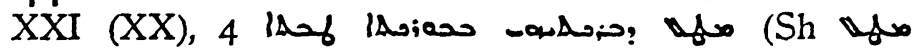
MNTXрнстос (= Lag ${ }^{\text {th }}$ : denn du bist ihm zuvorgekommen mit deinen besten Segnungen $=\mathrm{Lag}^{\mathrm{m}}=\mathrm{Sh}$ gegen Barh. : denn $d u$ hast ihn gesegnet mit gutem Segen).

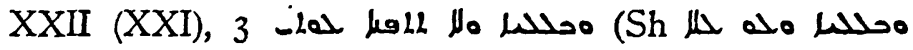

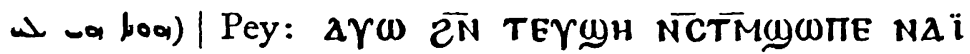
NоүмNтАөнт (= Lag ${ }^{\text {th }}$ : und in der Nacht soll es nicht werden mir Verstandlosigkeit $=$ Lag $^{\mathrm{m}}$ gegen Barh: und in der Nacht und nicht wendest du dich zu $\mathrm{mir}^{2}$ ).

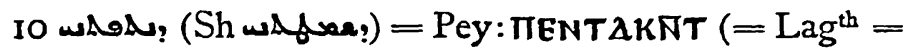
$\operatorname{Lag}^{\mathrm{m}}$ : der mich ziehen wird $\left.{ }^{3}\right)$.

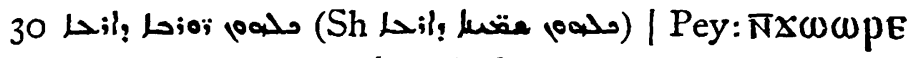

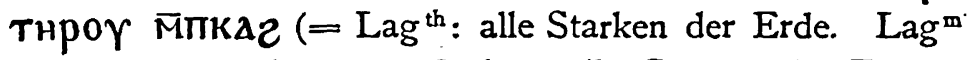
keniôut $=$ pinguis gegen Barh.: ,alle Grossen der Erde").

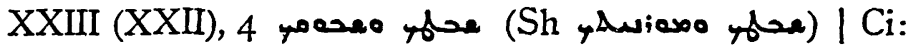

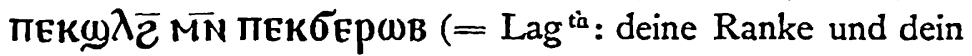
Zweig); Lag ${ }^{\mathrm{m}}$ : pekśbôt nem tekbaktêria (dein Stab und dein Stock4).

I Es ist fast so, als ob Barhebräus das nachfolgende $T \bar{B} B O L$ (= reinige mich) verlesen hätte in †CBWï (me docere); auch die Voranstellung des IA 1 würde damit stimmen.

2 Die Leseart des Barhebräus - lol holl لlo könnte möglicherweise Übersetzung von $\overline{N C T M} \bar{M} \omega \Pi \mathrm{N} \boldsymbol{N} i$ sein mit fehlerhafter Ableitung des $\omega \omega \Pi E$ von $\omega B$ convertere, permutare.

3 Die Worte des Aeg und der Sh sind Synonyma.

4 Die Leseart des Barhebräus stimmt eher mit Lagm als mit Ci 


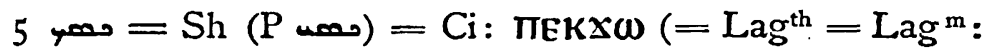
dein Becher).

XXIV (XXII), I awoas (Sh ald Wosoo) = Pey:

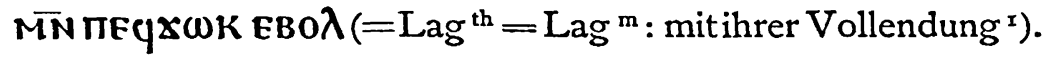

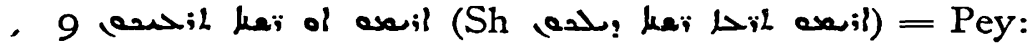

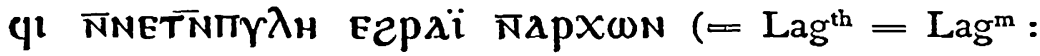
hebet euere Thore auf, Herrscher).

XXVI (XXV), 6 A TAKWTE ( $=\mathrm{Lag}^{\text {th }}=\mathrm{Lag}^{\mathrm{m}}$ : ich werde umgeben).

XXVII (XXVI), I -igor (= P; Sh -Loidando) = Pey: nAOYOEIN ( $=$ Lag $^{\text {th }}=\operatorname{Lag}^{\mathrm{m}}$ : mein Licht ${ }^{3}$ ).

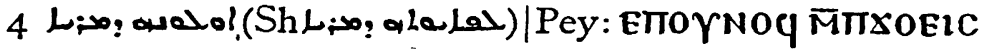
( $=$ Lag $^{\text {th }}=$ Lag $^{\mathrm{m}}$ : die Freude des Herrn; gegen Barhebr.: das Thal des Herrn 4 ).

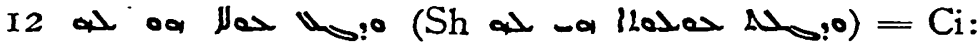

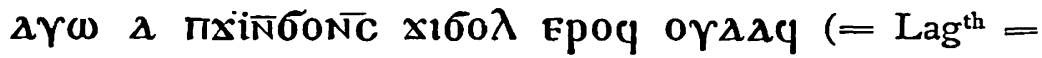
$\operatorname{Lag}^{\mathrm{m}}$ : und die Gewaltthätigkeit täuscht sich 5).

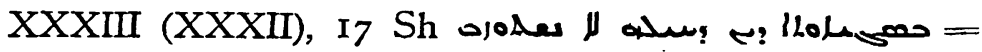
Aeg (P بasa) = Ci: NqNanoram

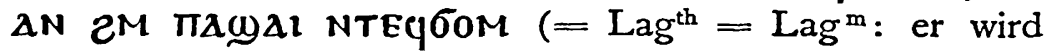
nicht gerettet durch die Grösse seiner Kraft).

und Lagth überein; denn for kann auch "Stab“ heissen, nicht aber kann Hoe in der Bedeutung "Ranke" gebraucht werden.

I Die beiden syrischen Worte sind hier wohl nur synonym verwendet.

2 Vergl. oben z. St.

3 Die beiden syrischen Worte wollen keinen verschiedenen Sinn wiedergeben (cf. die gleiche Stelle beim Armenier), wiewohl die Kopten für „Erleuchtung“ eine eigene Bildung (Epoץ(WINI) haben.

4 Barhebräus erklärt die Leseart damit, dass der Übersetzer den „Ort der Freude“ setzte.

5 Es ist aus dem Syrischen nicht klar, ob ل la neutral (= Frevel) oder masculinisch $\mathrm{zu}$ fassen ist (=Frevler); wahrscheinlich ist das erstere, sofern es sich auch hier, wie öfter, um synonyme. Bildungen (l) und llaveح) zu handeln scheint. 
XXXVI (XXXV), 7 lasl: liaf (= Sh; P und Arm ohne :) - Pey: त̈ der Weise der Berge Gottes).

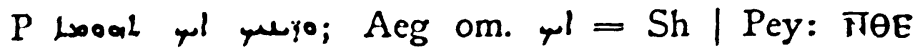
FTriorn ( - Lag $^{\text {th }}=\mathrm{Lag}^{\mathrm{m}}$ : wie der Abgrund, also gegen Barhebräus).

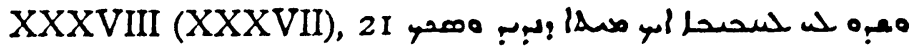
حمئi (bei Sh nur in einigen Hsn. ohne den letzten Zusatz)=

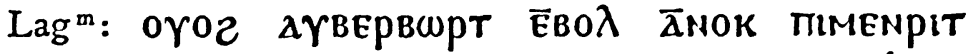

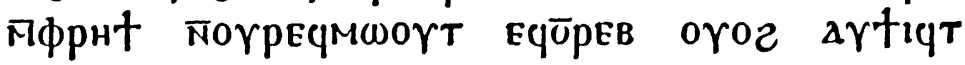
ETACAPz (und sie warfen mich, den Geliebten, hinaus in der Weise eines Toten, der verabscheut wird, und sie durchbohrten mein Fleisch ${ }^{\mathrm{I}}$ ); $\mathrm{Ci}$ hat, wie Sh, den Zusatz ebenfalls nicht.

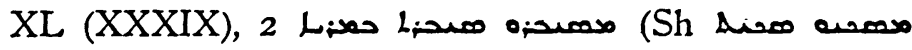

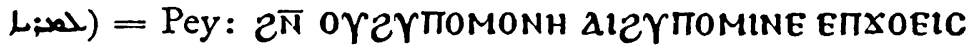
( - Lag $^{\text {th }}$ : in Erwartung wartete ich auf den Herrn); Lag $^{\mathrm{m}}$ hat nur statt des griechischen Wortes das koptische gusust. ${ }^{2}$

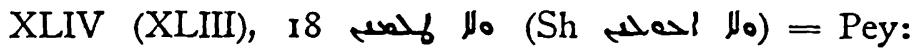

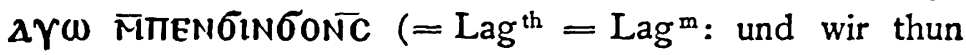
nicht Unrecht 3).

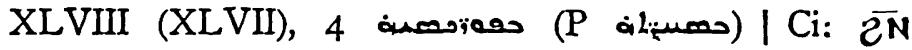
NECMA ETTAELHY (= Lag th: an ihren Orten, welche ehrwiirdig sind; $\operatorname{Lag}^{\mathrm{m}}$ : ken nê et horš $=$ welche schwer $\sin { }^{4}$ ).

I Die Stelle führt in koptischer Schrift $\mathrm{Ci}$ in den Anmerkungen an; damit stimmt Lagm überein.

2 Die beiden syrischen Worte wollen keinen verschiedenen Sinn besagen, sind vielmehr synonym.

3 Oben (Ps. XXVII, 12) war das gleiche Wort: бOÑC durch a (لحa) wiedergegeben; wenn es hier im Unterschied von Sh (ح) mit 16 gegeben wird, so ist klar, dass dies nur dann statthaben kann, wenn die Citate bereits syrisch vorlagen; denn aus dem Koptischen selbst war kein Grund zu entnehmen, warum eher ilf als a am Platze wäre.

4 Die thebanische und memphitische Version haben die griechische 
LXXIII (LXXII), 4 poufsel llowel tlo (Sh l;;0so

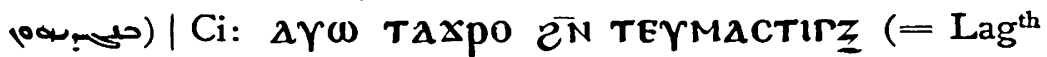
$=\mathrm{Lag}^{\mathrm{m}}$ : und Festigkeit in ihrer Geissel; gegen Barh.: und nicht Heilung in ihren Ruten ${ }^{x}$ ).

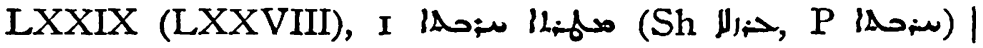

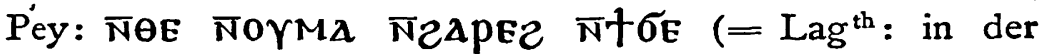
Weise eines Ortes des Bewachens der cucurbita; Lag ${ }^{\mathrm{m}}$ hat statt $\bar{N} \dagger \sigma \in$ das Wort $\boldsymbol{x i x i}$ ğiği $=$ fructus autumnalis, ò $\pi \omega \rho \alpha$ gegen Barh: zerstörte Wache ${ }^{2}$ ).

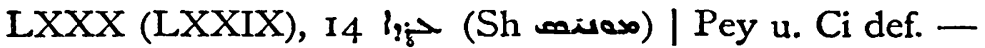

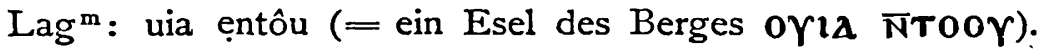

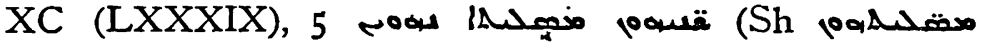

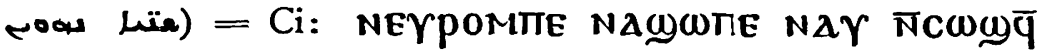
(ihre Jahre werden ihnen verachtet sein, Lagm lässt noch dazu NaY aus wie Berhebräus).

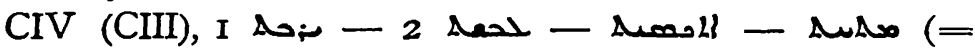
Sh, Arm, Nestor.; P hat statt der 2. sg die 3. Person sg). $\mid$

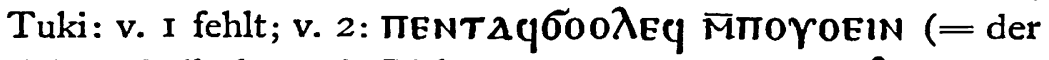

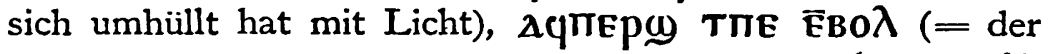

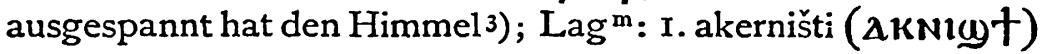
$=\mathrm{du}$ bist verherrlicht worden), aktêitu (АКтнıтO $=\mathrm{du}$

Vorlage wörtlich wiedergegeben; die Leseart des Barhebräus setzt eine andere griechische Vörlage voraus, etwa $\beta \alpha \sigma i \lambda \varepsilon i \alpha=$ Königsburg cf. Field, Hexaplorum quae supersunt II. ad h. l., wo ähnliche Varianten angegeben sind.

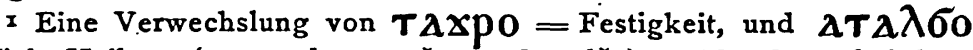
$=$ Nicht-Heilung (gesprochen : tağro und atalčo) aus Unachtsamkeit läge nicht ausser aller Wahrscheinlichkeit; ohnedem müsste eine selbständige Leseart bei Barhebräus gefunden werden.

2 Verwandte Auffassungen (acervus, acervus lapidum) finden sich bei Field 1. c. angegeben; wie jedoch die Lesung des Barhebräus entstanden sei, ist nicht klar; ZAPEZ klingt allerdings ziemlich an bPEB und $2 \omega \mathrm{pB}=0 ; \omega=$ desertus locus an.

$3 \mathrm{Da}$ die Lücken sahidisch nicht auszufüllen waren, so ist dieses Citat von Ciasca dem boheirischen Texte von Tuki (Rudimenta linguae copticae, Rom 1778) entnommen. 


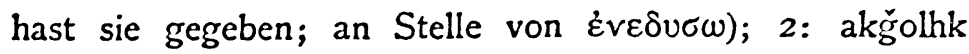
(= du hast dir angethan), afčolk (= er hat ausgespannt). Also stimmen die drei ersten Formen von Lag $^{\mathrm{m}}$ mit Barhebräus überein.

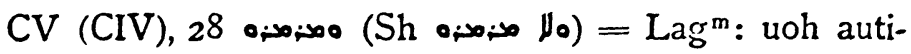
ǧônt $\left(\operatorname{A\gamma t\gamma \omega NT}=\right.$ und sie erbitterten $\left.{ }^{2}\right)$.

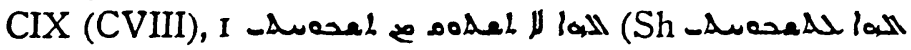

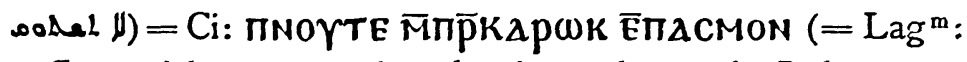
- Gott, nicht mögest du schweigen über mein Lob $^{2}$ ).

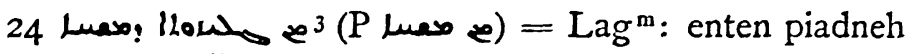
(= wegen der Öllosigkeit); jedoch Pey: ETBE TNNEZ (= wegen des Öles $=\mathrm{P}=\mathrm{LXX}$ ).

CXV, II (v 2 nach LXX) - (Sh -losaolsta) | Lag $^{\mathrm{m}}$ : ken patômt (in meinem Staunen $=\mathrm{Sh} ; \mathrm{Ci}$ und $\mathrm{Pey}$ def.; gegen Barhebräus: in meiner Unwissenheit4).

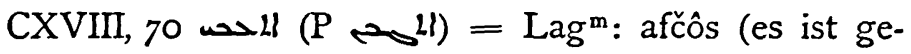
ronnen; Pey und $\mathrm{Ci}$ def.5).

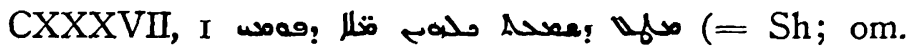
$\mathrm{P}, \mathrm{Hebr}, \mathrm{Arm})=\mathrm{Lag}^{\mathrm{m}}$ : ğe aksôtem enisaği têru ente rôi (denn du hast alle Worte meines Mundes gehört; $\mathrm{Ci}$ und Pey def.).

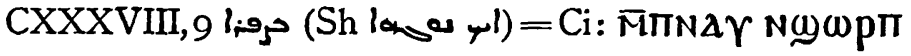

I Ci und Pey sind hier lückenhaft.

2 Die koptische Leseart: ETICMOY kann sowohl mit $\perp$ als mit $\odot$ wiedergegeben werden; die Differenz zwischen Sh und Aeg scheint deshalb schon syrisch vorhanden gewesen zu sein. - In der Stellung stimmt das Citat des Barhebräus genau mit dem koptischen Texte überein.

3 So die Ausgabe von Lagarde; S 326 bietet sel Lal.

4 Die Leseart des Barhebräus ist offenbar eine Verlesung: statt patômt = mein Erstaunen hat Barhebräus oder sein Gewāhrsmann gelesen: p- (=Artikel), at (= ohne, Negation) und emi (wissen): $=$ in meiner Unwissenheit.

5 kännte nur eine Variante sein, die den nämlichen Sinn wie $\$$ "l hat; wahrscheinlicher ist eine Verwechslung von $\omega_{\omega T}=$

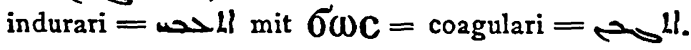


( $=$ in der Zeit der Morgenröte; Lag ${ }^{\mathrm{m}}$ hat blos $\overline{\mathrm{N}} \omega \omega \mathrm{\omega} \Pi$ $=$ "mane" und fügt hinzu, entatakôu eratu $=$ welche er aufgerichtet hat ${ }^{\mathrm{I}}$ ).

Die Summe der Citate aus der koptischen Bibelübersetzung beläuft sich auf 45 , also nur ein $1 / 3$ der armenischen Citate. Auch hier kehren ähnliche Fragen wieder, die wir uns oben gestellt haben. Zunächst finden wir den Schluss, den wir oben aus dem Namen auf die koptische Bibelübersetzung gezogen haben ${ }^{2}$, bestätigt. Aus denjenigen Stellen, wo Barhebräus mit Sh und mit den koptischen Übersetzungen übereinstimmt, ist nicht viel zu folgern. Wohl aber sprechen für die Richtigkeit obigen Schlusses Stellen wie 8, 5, 38, 21; 105, 28; 109, 24, wo Barhebräus gegen Sh mit einer der beiden zugänglichen koptischen Übersetzungen sich deckt; um so mehr sind diejenigen Citate hierfür zu verwerten, wo Barhebräus durch die beiden koptischen Texte gegen Sh und LXX bestätigt erscheint, wie 24, 9; 90, 5 ; 1 38, 9. Haben wir bei der Frage über die armenischen Citate den fehlerhaften Auffassungen des vorliegenden Textes eine ausschlaggebende Bedeutung beigemessen, so können wir hier nicht auf so zahlreiche Beobachtungen hinweisen; aber es ist auch dä nicht versäumt worden, auf mehr oder minder wahrscheinliche Verlesungen und sonstige Fehler hinzudeuten, die das Gepräge koptischen Ursprungs an sich tragen. Sicher scheint besonders die interessante Stelle I I5, II, wo sich das rätselhafte Citat so einfach als falsche Auffassung der koptischen Vorlage begreift.

Scheint demnach, alle diese Gründe zusammengenommen,

I Offenbar ist die Leseart von Lagm eine Combination der Varianten

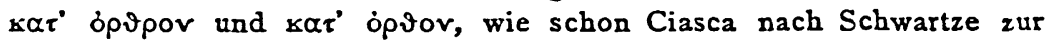
Stelle anfübrt.

2 Vergl. p. 128 n. 3 . 
der wirkliche koptische Ursprung dieser Barhebräuscitate festzustehen, so ist weiterhin zu untersuchen: welcher von den verschiedenen koptischen Übersetzungen, die wir kennen, schliesst sich die Vorlage des Barhebräus an: Die Frage nach der Zahl der koptischen Bibelübersetzungen hängt $a b$ von der Zahl der ehedem vorhandenen koptischen Dialekte, und die eine Frage ist noch so ungeklärt wie die andere ${ }^{\mathrm{x}}$ ). Oben wurden nur 2 Übersetzungen beigezogen, und darauf muss sich auch die Untersuchung über die koptischen Citate beschränken. Im sog. faijumischen und mittelägyptischen Dialekte existieren zwar verschiedene Fragmente, jedoch darunter nichts aus den Psalmen; dasselbe gilt von den achmîmischen Überresten einer Bibelübersetzung ${ }^{2}$. Ein koptisches Psalterium besitzen wir jedoch im boheirischen (früher memphitisch) und im sahidischen (früher thebanisch 3 ) Dialekte; in beiden Dialekten ist dasselbe auch bereits durch den Druck allgemein zugänglich gemacht, wie oben (S. 129 Anm. 5) angegeben. Leider sind jedoch die Ausgaben noch keineswegs hinreichend fundiert und kritisch genug bearbeitet, um mehr als provisorische Resultate aus einer Vergleichung mit ihnen zu erzielen.

Wenn wir nun obige Zusammenstellung überschauen, so unterliegt es keinem $Z$ weifel, dass die Barhebräuscitate sich viel näher an die boheirische (memphitische) Übersetzung anlehnen, als an die sahidische (thebanische). Wenn wir von der Gesamtsumme (45) der Citate die Stellen ab-

I Vergl. Hyvernat H., in Vigouroux, Dictionnaire de la Bible II, $93 \mathrm{I}-5 \mathrm{I}$; Nestle $\mathrm{E}$. in Urtext und Übersetzungen der Bibel, S. A. der PRE 3 p. $144 \mathrm{ff}$.

2 Vergl. Hyvernat in Revue biblique 1896, wo der Inhalt der einzelnen Handschriften genau verzeichnet ist.

3 Oben wurden für die Abkürzung und in den Erklärungen die früheren Bezeichnungen (memphitisch, thebanisch) zum Teil beibehalten, weil die benützten Ausgaben dieselben im Titel gebrauchen. 
ziehen, an denen Barhebräus mit Sh und den beiden Übersetzungen übereinstimmt $(6$, nämlich 7,$7 ; 23,5 ; 26,6$; $33,17 ; 36,7 ; 137,1)$, weiterhin diejenigen, an denen Barhebräus für beide Übersetzungen gegen Sh steht $(4: 24$, 9; 90,5 ; 105, 28; 1 38, 9) - 9 Stellen kennzeichnen sich blos als syrische Varianten ohne verschiedene koptische Vorlage - so ist wohl von einer bemerkenswerten Annäherung an die boheirische Übersetzung gegenüber der sahidischen zu reden, wenn 5 Stellen $(5,9 ; 8,5 ; 23,4 ; 38,21 ; 109,24)$ ausdrücklich mit der boheirischen Übersetzung übereinstimmen. Besonders $\mathrm{zu}$ betonen ist die Gemeinsamkeit einer Glosse $\mathrm{zu}$ 38, 21, die sich sonst nirgends findet als in der boheirischen Übersetzung und dem entsprechenden Citate des Barhebräus ${ }^{x}$. Diese auffallige Übereinstimmung möchte verleiten, in der boheirischen Übersetzung ohne Weiteres die Vorlage der Barhebräuscitate zu suchen. Trotzdem zeigen auch diese Bruchstücke eine gewisse Selbständigkeit. Manche Varianten, die Barhebräus bietet, mögen sich ja als fehlerhafte Auffassungen bei mangelhafter Kenntnis der koptischen Sprache oder oberflächlicher Einsichtnahme in den Text erklären lassen, so etwa 16,$11 ; 17,14 ;(118,70)$; II 5 , II. Bei manchen selbständig scheinenden Lesungen (z. B. 17,$7 ; 18,40.44 ; 19,13 ; 22,3.30 ; 27,4 ; 73,4 ; 79,1$; 80 , 14) mag es nicht ausgeschlossen sein, dass bei besserer Kenntnis der koptischen Sprache oder glücklicherer Combinationsgabe ein anderer einen fehlerhaften Ursprung derselben noch aufdecken kann. Sicher jedoch sind als selbständige Lesearten bei dem Kopten des Barhebräus zu betrachten Stellen wie 17, 15; 18, 26 (bis); 20, 10; 21, 4; 48, 4. Diese Stellen legen eine gewisse Reserve auf, die boheirische Übersetzung und den Kopten des Barhebräus

I Auch in Field, Hexaplorum quae supersunt II. ad h. 1 . ist keine àhnliche Leseart angegeben. 
$z u$ identificieren. Mehr zu sagen, etwa beide für verschieden zu erklären, dazu sind aber die Stellen, die in Frage kommen, doch $\mathrm{zu}$ wenig. Solche Varianten gehen wohl über die Verschiedenheiten unter Handschriften derselben Übersetzung nicht hinaus. Diese Möglichkeit ist um so mehr offen zu halten, wenn wir noch einen äusseren Umstand beachten: eine Verbindung und ein Austausch zwischen Ägypten und Syrien musste in erster Linie nicht von Oberägypten, sondern von Unterägypten ausgehen und auf dieses und die Meergegend treffen, also über den Bereich hin sich vollziehen, wo dieboheirische Bibelübersetzung in Gebrauch war.

Hat nun Barhebräus die koptische Übersetzung selbst eingesehen oder sind ihm die Citate bereits syrisch vorgelegen? Es wird das gleiche Verhältnis zu erwarten sein, wie bei den syro-armenischen Citaten. Bestätigt wird diese Annahme durch die zahlreichen koptischen Citate, die nur syrische Varianten bilden, keineswegs einen von den übrigen Übersetzungen verschiedenen Sinn feststellen wollen (z. B. 7,$7 ; 18,43 ; 22$, 10; 24, I; 27, I. I $\left.2 ; 40,2 ; 44,18 ; 109, I^{1}\right)$. Auch hier ist wohl nicht anzunehmen, dass eine vollständige syrische Übersetzung aus dem Koptischen vorhanden gewesen sei; sondern die Citate werden als Randlesungen zu irgend welchen syrischen Handschriften vorgefunden worden sein.

Eine weitere Untersuchung, welche Bedeutung allenfalls den Citaten des Barhebräus für die Kritik des koptischen Bibeltextes und weiterhin der vorgelegenen griechischen Handschriften zukomme, ist aussichtslos, weil der Stellen zu wenige sind.

I Vergl. besonders die Note zu 44, 18. 
Gering mag die Förderung sein, welche die biblische Textkritik aus diesen syro-armenischen und syro-koptischen Citaten des Barhebräus zieht. Aber sicher gewinnt das Bild eines Schriftstellers einige günstige Züge, der in so ausgedehnter Weise textkritisches Material herbeischaffte und zur Fundierung des Schrifttextes verwandte. Rege muss auch das wissenschaftliche Leben beim syrischen Volke gewesen sein, wenn die Trennung durch Sprache und Entfernung kein Hindernis schuf, den gegenseitigen geistigen Verkehr zu hemmen. 